\title{
Urban Road Network Emergency: An Integrative Vulnerability Identification Method
}

\author{
Huaikun Xiang (iD \\ School of Automotive \& Transportation Engineering, Shenzhen Polytechnic, Shenzhen 518055, China \\ Correspondence should be addressed to Huaikun Xiang; xianghuaikun@szpt.edu.cn
}

Received 2 May 2021; Accepted 30 May 2021; Published 11 June 2021

Academic Editor: Long Wang

Copyright (c) 2021 Huaikun Xiang. This is an open access article distributed under the Creative Commons Attribution License, which permits unrestricted use, distribution, and reproduction in any medium, provided the original work is properly cited.

\begin{abstract}
The vulnerability of an urban road network is affected by many factors, such as internal road network layout, network structure strength, and external destructive events, which have great uncertainty and complexity. Thus, there is still no unified and definite vulnerability analysis scheme available to cities. This paper proposes an integrative vulnerability identification method for urban road networks, which mainly relates to the vulnerability connotation and characteristics analysis of urban road networks during emergency, and vulnerability comprehensive evaluation indices design based on urban road network connectivity, traffic efficiency and performance, and an empirical study on a vulnerability identification method of an urban road network. In the empirical case, a real road network and traffic operation data were used from Science and Technology Park of Shenzhen City, China. In the context of one certain emergency scenario, the stated preference survey method and maximum likelihood method are used to solve the road users' random travel choice behavior parameters; subsequently, based on the traffic equilibrium distribution prediction, the traffic vulnerability identification methods of the road network in this region were verified before and after the emergency. The method presented here not only considers the impact of network topology changes on road network traffic function during emergency but also considers the impact of dynamic changes in road network traffic demand on vulnerability; therefore, it is closer to the actual distribution of urban road network traffic vulnerability.
\end{abstract}

\section{Introduction}

The urban road traffic system is called the "lifeline" system of a city, which plays a key role in the daily commuting, logistics, and business travel of the city as discussed by various sources [1-3]. However, all kinds of traffic accidents, road failures (such as landslides or flash floods), or bad weather (such as rainstorms, fog, or blizzards) often create significantly adverse effects on the normal operation of urban transportation network. Under the impact of various emergencies, the vulnerability of urban road network (VURN) is constantly exposed. Some vulnerable links in the network not only lead to the delay of network travel time but also cause cascading failure or an avalanche effect of the whole road network due to interruption and congestion. The American Road and Transportation Builders Association points out that nearly $75 \%$ of freight transportation in the United States is carried out through vulnerable links (such as highways and traffic bottlenecks), which cause approximately 243 million hours of delay for truck drivers every year $[4,5]$. In this context, the VURN is regarded as an important research topic [6].

The VURN's main research purpose is to obtain a method that can quickly, accurately, and reliably identify those vulnerable links in the road network, to provide assistance to reduce the losses caused by various emergencies on vulnerable roads $[7,8]$, and fundamentally provide decision support for improving the risk resistance ability of urban road networks. Usually, vulnerability analysis of the road network is carried out on road topology network models based on network theory. Generally, the method of gradually moving some nodes or connecting edges in the road network is adopted to calculate and compare the changes of connectivity or traffic efficiency indexes of the whole road network before and after the change of road network structure; accordingly, the nodes or connecting 
edges which calculation results vary greatly are defined as the vulnerable parts of the road network. Usually, the performance parameters of complex topological network are selected as the connectivity index or traffic efficiency index of the road network, such as the degree, betweenness centrality, community structure, and cluster coefficient [9-17]. Based on these indicators to identify the VURN, although the results can identify the vulnerable part of the network to a certain extent, there are obvious defects in the practical application of the identification results. The main problem is that it cannot reflect the VURN and change the state of vulnerability [11]. Research shows that the public network with statistical significance may show completely different network characteristics [18].

Due to carrying various complex traffic and transportation activities, urban road networks will be impacted to varying degrees in addition to the topological structure changes of the road network after an emergency, which introduces difficulties to the vulnerability identification of the whole road network. The key problem is how to accurately, effectively, and reliably define the quantitative relationship between various traffic activities and the VURN. In this case, scholars have proposed a series of vulnerability evaluation indicators to reflect the traffic changes of a road network, such as the travel time based on the network robustness index $[19,20]$. Based on the analysis of the connotation and characteristics of the VURN, this paper proposes an integrative identification index which can comprehensively reflect the changes of urban road network vulnerability and designs the corresponding identification model and algorithm. The main contributions are as follows:

(1) A three-dimensional model for vulnerability analysis of an urban road network under emergency is established. The model can better explain the concept of the VURN, which mainly involves three variables, namely, the process variable of emergency, the traffic loss variable of the road network caused by the emergency, and the probability variable of the emergency.

(2) An integrative vulnerability identification index system of an urban road network is established. The VURN is affected by many factors, not only in the connectivity and stability of the network topology, but also in the effectiveness and reliability of the network service function, which are related to the process of emergency. Therefore, based on the comprehensive identification index system of the emergency occurrence process, the city can more accurately identify the VURN.

(3) The traffic demand is included in the vulnerability identification process. The comprehensive vulnerability identification model is constructed before and after the emergency, and the corresponding efficient algorithm is designed to identify the VURN.

The vulnerability identification method of an urban road network based on a comprehensive identification index system proposed here can reflect the vulnerability of a road network more comprehensively, so that it can identify those key locations and sections more accurately. The remainder of the paper is organized as follows: a conceptual and characteristic framework for vulnerability analysis is proposed. An integrative vulnerability identification method of the VURN during emergency is discussed, which mainly includes the design of the vulnerability index system, the improvement of the network traffic impedance function, and network traffic assignment modeling and model solving algorithm design. The proposed vulnerability identification method based on actual road network traffic survey data is verified and analyzed. The results of urban road network vulnerability discrimination under different index combinations are analyzed.

\section{Related Work}

Early studies of vulnerability emerged in the field of engineering and were then extended to the social economic and political institutional level by social scientists. In research related to road network disruption, efforts were made to define the vulnerability of road network [6, 21, 22]. Since then, several studies have attempted to provide methods for evaluating network performance in terms of vulnerability or robustness [20, 23-27]. In fact, the vulnerability assessment method of urban road networks is closely related to the vulnerability index, and this is related to the understanding of the concept of vulnerability. Therefore, the cognition of the concept directly determines the design of an urban road network vulnerability assessment method. The following literature review focuses on the relevant research results on urban road network vulnerability in recent years.

To date, there is no definite and unified definition of the concept of road network vulnerability [28]. Berdica [6] discussed the concept and considered that the vulnerability of a road network is sensitive to the time when the service capacity has declined sharply. Holmgren [29] believes that vulnerability should comprise the sensitivity of road networks to disaster risk. Husdal [30] considers that "vulnerability describes the nonoperability of the networks under varying strenuous conditions (i.e., the susceptibility to fail to function)". Taylor et al. [31] believe that if a small number of road sections in the network fail or significantly degrade the accessibility of nodes, then the nodes of the network are fragile; and if the failure of a certain road section will likely reduce the accessibility of the whole or some nodes of the network, then this road section is considered critical.

Erath et al. [32] studied the VURN in Switzerland. They defined the vulnerability of road network as the product of the probability of road failure under dangerous conditions and the sum of direct and indirect consequences caused by interruption. Jenelius et al. [33] believe that road network vulnerability can be defined as the study of the potential degradation of the road transport system and its impact on society; hence, risk theory can be used to measure the vulnerability of a road network. According to Yin et al. [15], the issues of vulnerability are "which areas in the road transportation system are easy to interrupt" and "which connections are the most critical to the operation of the 
whole system." Therefore, the vulnerability of a road network can be regarded as the integration of the possibility of road section failure and the potential impact of failure. Yang et al. [34] believe that the vulnerability of a road traffic network is that some units (including nodes and road sections) in the network are damaged or have failed due to the disruption of emergency situations, through the interaction between road network units and the continuous influence of the outside world. This type of loss or failure transfers among other units in varying degrees and finally contributes to the loss measurement of the whole road traffic network.

Emergency can be natural or man-made damage events, or small-scale events, such as explosion or fire in buildings, or large-scale damage events, such as an earthquake, radiation accident, bombing, or dangerous weather conditions in cities or areas [35]. The International Strategy for Disaster Reduction (UNISDR) defined a disaster as the negative effects of hazards on vulnerable socioeconomic systems, where vulnerability limits the coping capability to the impact of the hazard [36]. Emergencies are characterized by strong randomness, wide coverage, and a significant negative diffusion effect [37]. Once emergencies occur, it will be in a state of rapid diffusion initially. Once this state affects the urban transportation activities, its impact on urban road network traffic begins $[28,38,39]$. After a period of development and change, the impact on road traffic will reach a maximum, mainly manifested in the effect on road traffic infrastructure, the interruption of traffic flow operation, and the impact of travel demand fluctuation. When the emergency is finally under control, the impact ends. From this point forward, road traffic will enter the process of gradual recovery. For different road traffic networks, the recovery time varies. A short recovery time indicates that the road traffic network is more robust, and conversely, it indicates that the network is more vulnerable [40].

The connotation of road traffic network vulnerability should include the following characteristics: (1) vulnerability is an inherent attribute existed recessively when there is an emergency and is present in a road traffic network. (2) When encountering emergency, the inherent vulnerability of a road traffic network emerges. If the road traffic network is more prone to emergency situations, the inherent vulnerability is greater. (3) In examining the impact of emergency, if the loss degree of the road traffic network is more serious, it indicates that the inherent vulnerability is greater and that the road network is more vulnerable to external interference factors. (4) Once the emergency and losses are under control, if the service level of the road traffic network is conducive to effective restoration under external repair, the impact of the vulnerability is considered low; conversely, the impact is categorized as high. These characteristics of road traffic network vulnerability are shown in the concept map of a three-dimensional model as shown in Figure 1.

Figure 1 shows a three-dimensional coordinate system composed of probability, time, and loss, where $t_{s}$ is the start time of the emergency, $t_{m}$ is the time when the impact of the event reaches the maximum, and $t_{e}$ is the impact end time. The time period from $t_{s}$ to $t_{m}$ is called the influence period, in which the emergency will have a significant impact on the road traffic infrastructure, traffic operation, and the choice behavior of residential travel. The impact of the infrastructure and traffic operation mode has the most direct effect, and the response is also the fastest and easy to be spread, which is the most active and easily changed element in the whole system. Due to the transmission of information takes time, there is no way to quickly inform road users of a potential road block. This contributes to further unnecessary congestion in the emergency zone.

The period from $t_{m}$ to $t_{e}$ is called the recovery interval. Owing to the superposition of various influences, the recovery of a road traffic network needs a period. $P_{\max }$ is the maximum probability of an emergency, and $l_{\max }$ is the maximum loss caused by an emergency. Therefore, we define the vulnerability of a road traffic network as follows: the vulnerability of a road traffic network refers to the comprehensive embodiment of the probability of a road traffic network being affected, the severity of loss consequences, and the difficulty of recovery in an emergency encounter.

\section{Materials and Methods}

3.1. Index Design. The VURN is the result of a variety of complex, different, and uncertain factors, such as road conditions, network structure, traffic flow state, rescue point setting, resource scheduling, and repair strategy. When a serious traffic accident occurs, some units in the road network will be damaged or even invalid due to the situation. Simultaneously, the urban transportation department takes measures to ensure smooth continuity of the road network and conducts emergency rescue work to address the incident. If the road network is continuously affected by emergency, multiple types of damage can occur and spread impacting the road network. The transmission of road network vulnerability between road network units is triggered with the function failure of road network units [38], resulting in the so-called "avalanche effect," which creates significant difficulties for emergency rescue. This continues until the road network function returns to normal and the network is stabilized. Figure 2 expresses the road traffic flow distribution of this process as a schematic diagram.

In Figure 2, $t_{s}$ represents the starting time of the event, and the number of vehicles on the road is $x\left(t_{s}\right)$. After the incident, the road is interrupted, as it is not possible to share the information with every vehicle on the road in a timely manner, and because the vehicles entering the section have no choice, the inflow rate of vehicles in the accident section exceeds the outflow rate, until the accident is managed; that is, entering time point $t_{m}$, the number of vehicles on the road is $x\left(t_{m}\right)$. Starting from $t_{m}$, the outflow rate of vehicles on the road will be slightly higher than the inflow rate until the original capacity of the road is fully restored. This is time $t_{e}$, and the number of vehicles on the road is $x\left(t_{e}\right)$. Under ideal conditions, $x\left(t_{s}\right)$ is approximately equal to $x\left(t_{e}\right)$.

The VURN reflects the degree to which the network and network units are vulnerable to various interference factors. The more sensitive the network is to interference factors, the more vulnerable the parts are to the impact [28]. According 
to the previous analysis, after the road network suffers from emergency, the road network structure will be affected to varying degrees, and the traffic operation will be disturbed, which will be reflected in the loss degree of the whole network [41, 42], specifically in the changes of network connectivity [43], operation timeliness [44], service level, etc. Therefore, based on the influence degree of interference factors, this study designs a comprehensive identification index system of urban road network vulnerability under emergency conditions.

Depending on the severity of the situation, once an emergency has occurred, the topology of the original road network may significantly change, and this manifests in the node failure caused by interruption to traffic and sudden congestion at the intersection, thus affecting the connectivity of the road network.

3.1.1. Connectivity of Road Network and Its Change Measure in Case of Emergency. In this study, the road network connectivity $G$ is defined as the ratio of the actual number of edges to the maximum number of edges in the network. For a network node, the greater the change of connectivity before and after failure, the more significant the impact of the node is on the robustness of the road network, and the same is true for the connected edges of the network. The calculation formula of network connectivity is as follows:

$$
G=\frac{D}{3 V_{d}-6}
$$

where $D$ is the number of edges in the network and $V_{d}\left(V_{d}>3\right)$ is the number of nodes in the network. When the point fails, the actual number of edges $D^{\prime}$ of the road network after the failure is counted, and the corresponding connectivity $G^{\prime}$ and the change $\Delta G$ of the road network connectivity before and after the failure are calculated:

$$
\Delta G=\left|G-G^{\prime}\right|=\left|\frac{D}{3 V_{d}-6}-\frac{D^{\prime}}{3\left(V_{d}-1\right)-6}\right| .
$$

3.1.2. Network Efficiency and Its Change Measure during Emergency. In the event of emergency, the sudden congestion of the road section leads to the failure of a link, which affects the efficiency of the network traffic. In this paper, the mean value of the edge betweenness efficiency of the topological network is defined as the network efficiency under emergency. Research shows that the greater the network efficiency change rate before and after failure, the more significant the impact of the road section on road network robustness [41]. The calculation formula of network efficiency $E$ is as follows:

$$
E=\frac{\sum_{k_{1}, k_{2} \in N, k_{1} \neq k_{2}}\left(1 / l_{k_{1} k_{2}}\right)}{n(n-1)}, \quad(n>1),
$$

where $N$ is the node set, $n$ is the total number of road network nodes, and $l_{k_{1} k_{2}}$ and $l_{k_{1} k_{2}}^{\prime}$ are the shortest paths between the connection nodes $k_{1}$ and $k_{2}$ before and after the failure. When an edge fails, count and calculate the actual number of edge betweenness and the corresponding network efficiency after the failure and calculate the change of network efficiency $\Delta E$ before and after the edge failure according to formula (3):

$$
\Delta E=\left|\frac{\sum_{k_{1}, k_{2} \in N, k_{1} \neq k_{2}}\left(1 / l_{k_{1} k_{2}}\right)}{n(n-1)}-\frac{\sum_{k_{1}, k_{2} \in N, k_{1} \neq k_{2}}\left(1 / l_{k_{1} k_{2}}^{\prime}\right)}{n(n-1)}\right| .
$$

3.1.3. Road Network Traffic Performance and Its Change Measure during Emergency. Considering that the urban road network is facing the impact of emergency, besides the change of the structure performance, the traffic operation of the road network will also be directly affected. Therefore, this study designs a comprehensive vulnerability measurement index which is more consistent with the actual situation of urban road traffic. Let $C_{i}$ denote the overall loss of the road network after unit $i$ in the road network is impeded by an emergency, also known as path impedance, $I$ denotes the number of road network units, $K$ denotes the number of failure units in this case, $k$ denotes the $k^{\text {th }}$ failure unit (road section or intersection) in the road network, and $t(x)_{k}$ denotes the loss of unit $k$ under traffic operation condition $x$, and it is as follows:

$$
C_{i}=\sum_{k=1}^{K} t(x)_{k}, \quad k=1,2, \ldots, K, i=1,2, \ldots, I .
$$

In the formula, $t(x)_{k}$ is related to the emergency rescue technology $R_{k}$ at $k$ and the failure loss of the upstream failure unit. For the specific calculation method, see the theoretical derivation in "Traffic Analysis during Emergency" in Section 3.2. Here, the final loss of road network users is selected as the measure. As the unit loss propagates in turn, $k-1$ represents the nearest upstream failure unit $k$, so $t(x)_{k}=$ $f\left(t(x)_{k-1}, R_{k}\right)$ is obtained. The final vulnerability of the road network is caused by the sudden impedance of the road network unit to measure the maximum road network loss of the project:

$$
L=\max C_{i}, \quad i=1,2, \ldots, I .
$$

In the above formula, $L$ is the indicator of traffic VURN. $\Delta L=\left|L-L^{\prime}\right|$ is the change of total loss before and after the emergency.

3.1.4. Integrated Identification Index of Urban Road Network Traffic Vulnerability during Emergency. From the previous analysis of urban road traffic network vulnerability, we can observe that the VURN is mainly characterized by its own structure and traffic function. Therefore, based on a summary of existing research results, this study selects three key index parameters to construct the traffic vulnerability identification index of the urban road network, which include the connectivity index, the traffic efficiency index, and the traffic performance index. However, these three indicators only judge the VURN from different aspects. In order to judge the VURN more accurately and comprehensively, it 
is necessary to develop a more comprehensive identification index based on these three vulnerability indicators.

First, the three subindicators are standardized without dimension, so that the three subindicators of vulnerability after data standardization are $\left\{\Delta G^{\prime}, \Delta E^{\prime}, \Delta L^{\prime} \mid \in(0,1)\right\}$. On this basis, based on the weighted method, the comprehensive identification index of vulnerability $v$ under an emergency is constructed as follows:

$$
v=\varepsilon \Delta G^{\prime}+\varnothing \Delta E^{\prime}+\gamma \Delta L^{\prime} .
$$

In the above formula, $\varepsilon, \varnothing$, and $\gamma$ represent the weight factors of the road network connectivity index, traffic efficiency index, and urban road network traffic performance index, respectively, where $\varepsilon, \varnothing$, and $\gamma$ are less than 1 , and $\varepsilon+\varnothing+\gamma=1$. Because it is difficult to determine the correlation between the three vulnerability subevaluation indexes objectively and quantitatively, this study uses the classic Delphi method [45] which involves collecting industry expert scores to determine the weight factor. Simultaneously, according to the Delphi method to determine the comprehensive level of urban road network traffic vulnerability under emergency situations, it is divided into five levels, including very low (Level 1), low (Level 2), medium (Level 3), high (Level 4), and very high (Level 5). The specific scale is shown in Table 1.

\subsection{Traffic Analysis during Emergency}

\subsubsection{Road Network Impedance during Emergency}

(1) Section Impedance. When an emergency occurs, if different types of travelers (a total of D) have uncertain estimation bias for the travel time of the road section in the traffic network, the estimated cost $T_{a}^{d}$ of the class $d$ travelers for the road section $a$ can be expressed as follows:

$$
T_{a}^{d}=t_{a}\left(x_{a}\right)+\varepsilon_{a}^{d}, \quad \forall a, d,
$$

where $x_{a}$ is the flow of segment $a$ and $t_{a}\left(x_{a}\right)$ is the cost determined by segment $a . t_{a}\left(x_{a}\right)$ adopts the road resistance calculation formula which reflects the road traffic operation characteristics under emergency situations derived from the Bureau of Public Road (BPR) function of the United States:

$$
\begin{aligned}
& t_{a}=t_{a}^{0}\left[1+\boldsymbol{\alpha}\left(y_{a}\right)^{\beta}\right], \\
& y_{a}= \begin{cases}\frac{x_{a}}{c_{a}} & y_{a} \in[0,1], \\
\frac{\left(2 c_{a}-x_{a}\right),}{c_{a}} & y_{a} \in[1,2] .\end{cases}
\end{aligned}
$$

In the above formula, $t_{a}^{0}$ is the driving time of the road section between two intersections when the traffic volume is 0 , also known as zero flow cost; $c_{a}$ is the actual traffic capacity (vehicle/h) of road section $a$; and $\boldsymbol{\alpha}$ and $\boldsymbol{\beta}$ are the undetermined parameters of the model, with the general values of 0.15 and 4, respectively [45], which can also be calibrated according to the measured data [46]. The relationship between impedance and traffic flow is increasing monotonically, which reflects the traffic congestion effect under emergency situations. $\varepsilon_{a}^{d}$ is the random deviation of class $d$ travelers to Section $a$, and its mathematical expectation is $E\left[\varepsilon_{a}^{d}\right]=0$. Therefore, the estimated cost $T_{a}^{d}$ of the link impedance is a random variable, and $E\left[T_{a}^{d}\right]=t_{a}, \forall a, d$.

(2) Path Impedance. In the urban road network $G(V, E)$, there is an effective path set $K_{r s}$ between all the OD (origin destination) pairs $r s$, and then the cost estimate $C_{d, k}^{r s}$ of the $d$ class traveler for the $k^{\text {th }}$ path in the road network is

$$
C_{d, k}^{r s}=\sum_{a} T_{a}^{d} \boldsymbol{\delta}_{a, k}^{r s}, \quad \forall r, s, d ; k \in K_{r s},
$$

where $\boldsymbol{\delta}_{a, k}^{r s}$ is the correlation coefficient between link $a$ and path $k$. If link $a$ is on the $k^{\text {th }}$ path between $r s$, then $\boldsymbol{\delta}_{a, k}^{r s}=1$; otherwise, $\boldsymbol{\delta}_{a, k}^{r s}=0$. Similarly, according to the accumulation of random variables, $C_{d, k}^{r s}$ is also a random variable, and $E\left[C_{d, k}^{r s}\right]=c_{d, k}^{r s}$, that is,

$$
c_{d, k}^{r s}=\sum_{a} t_{a} \boldsymbol{\delta}_{a, k}^{r s}, \quad \forall r, s, d ; k \in K_{r s},
$$

where $c_{d, k}^{r s}$ is the mathematical expectation of the random variable $C_{d, k}^{r s}$. From the above analysis, the path impedance $C_{d, k}^{r s}$ not only reflects the road traffic operation state of each road section but also has the monotonous change characteristics, which can ensure the calculation needs of the unique solution of the stochastic user equilibrium assignment model.

3.2.2. Travel Choice during Emergency. In case of emergency, each traveler will make their own choice on the travel path and travel mode according to their own specific conditions. Each path has an equal probability of being selected [47]. According to the Wardrop traffic equilibrium principle, the probability $P_{d, k}^{r s}$ of the class $d$ traveler selecting the route $k$ among $r s$ is the probability that the estimated cost (road impedance) is the probability that the estimated cost (road impedance) is the least on all possible paths between the origin and destination (OD) pairs, namely,

$$
P_{d, k}^{r s}=P\left(c_{d, k}^{r s} \leq c_{d, l}^{r s}, \forall l \neq k\right), \quad \forall r, s, d ; k \in K_{r s} .
$$

The above formula is calibrated according to the logit model [48], and the specific form of $P_{d, k}^{r s}$ can be obtained as follows:

$$
P_{d, k}^{r s}=\frac{\exp \left(-\theta_{d} c_{d, k}^{r s}\right)}{\sum_{l \in K_{r s}} \exp \left(-\theta_{d} c_{d, l}^{r s}\right)}, \quad \forall k \in K_{r s},
$$

where $a$ is the parameter to be calibrated in the logit model for class $P_{d, k}^{r s}$ travelers.

\subsubsection{Road Network Traffic Distribution during Emergency.} Considering the behavior of travelers' route selection in emergency situations, the traffic flow $f_{d, k}^{r s}$ of the $k^{\text {th }}$ route in one of OD pair $r s$ and OD demand $q_{r s}$ can meet the following conditions: 


$$
\left\{\begin{array}{l}
f_{d, k}^{r s}=q_{r s} u_{d}^{r s} P_{d, k}^{r s}, \\
\sum_{d} u_{d}^{r s}=1,
\end{array}\right.
$$

where $u_{d}^{r s}$ is the proportion of type $d^{\text {th }}$ travelers in OD pair $r s$.

From the above model, under the premise that $q_{r s}$ and $u_{d}^{r s}$ are known, the path flow $f_{d, k}^{r s}$ is related to the path selection probability $P_{d, k}^{r s}$, while $P_{d, k}^{r s}$ is determined by the parameter $\theta_{d}$ and the estimated value of path impedance $c_{d, k}^{r s}$, which is affected by the estimated value of travel time $T_{a}^{d}$, where $T_{a}^{d}$ is the function of flow $x_{a}$; therefore, continuous selection can ensure that the network traffic finally reaches the user equilibrium state. The user equilibrium assignment model is given as follows [45]:

$$
\begin{aligned}
& \min Z(f)= \sum_{d} \sum_{r} \sum_{s} \sum_{k \in K_{r s}} \frac{1}{\theta_{d}} f_{d, k}^{r s} \ln f_{d, k}^{r s} \\
&+\sum_{a} \int_{0}^{x_{a}} t_{a}(w) \mathrm{d} w, \\
& \text { s.t. } x_{a}= \sum_{d} \sum_{r} \sum_{s} \sum_{k \in K_{r s}} f_{d, k}^{r s} \delta_{d, k}^{r s}, \\
& \sum_{d} \sum_{k \in K_{r s}} f_{d, k}^{r s}= \sum_{d} q_{r s} u_{d}^{r s} P_{d, k}^{r s}, \\
& \sum_{d} u_{d}^{r s}= 1, \\
& f_{d, k}^{r s} \geq 0 .
\end{aligned}
$$

The above equations (16)-(19) are road flow constraint, path flow constraint, traveler proportion constraint, and nonnegative flow constraint, respectively, in which different travelers type $d$, proportion $u_{d}^{r s}$, and route selection probability $P_{d, k}^{r s}$ are considered. Obviously, when $q_{r s}, u_{d}^{r s}, \theta_{d}$, and other parameters are known, the objective function of the model is strictly convex with respect to $f_{d, k}^{r s}$, and the constraints are linear, so it has a unique path flow solution.

Further analysis shows that parameter $\theta_{d}$ has the random characteristics of the whole model. When $\theta_{d} \longrightarrow \infty$ is satisfied, the objective function becomes a standard user's equilibrium (UE) problem. When $\theta_{d} \longrightarrow 0$ is satisfied, OD demand matrix $\left[q_{r s}\right]$ will be distributed evenly on the road network, and all path costs will be equal.

\subsubsection{Design of Comprehensive Vulnerability Identification} Algorithm. Under the impact of emergency situations, there is a sudden impact on road traffic at the beginning of the event, and subsequently to the traffic demand changes caused by road users' travel choice behavior after information diffusion, so that the traffic flow of each OD trip in the road network is redistributed. In this process, the traffic impedance and its induced travel efficiency will be highlighted, so that the extent of the road network vulnerability can be identified here. Based on the previous theoretical analysis and modeling, the vulnerability identification algorithm is described as follows:

Step 1: for the selected road network, the design and investigation plan shall be carried out: (i) SP investigation of travel selection behavior in the absence of emergency and (ii) in the case of emergency. Based on the survey data and the principle of 3.3 , the parameters of the logit model are calibrated to obtain the trip selection probability of all kinds of type $d$ travelers in the event of emergency.

Step 2: for the road network $G(V, E)$, according to the principle of 3.4 , the demand $q_{r s}$ of OD pair $r s$ is allocated to the network using the random average assignment method, and the traffic volume $x_{i}(i \in L)$ of each road section is obtained;

Step 3: according to the principle of 3.2, calculate the impedance $T_{a}^{d}$ and path impedance $c_{d, k}^{r s}$ of each road section, and then calculate the operation efficiency of the road network before the emergency according to equations (1)-(7).

Step 4: after an emergency occurs, the topological structure of the road network is broken down one by one according to its impact on nodes (intersections) and road sections, and then the path impedance of each road section is calculated step by step; finally, the maximum road network loss caused by the emergency is calculated according to equations (1)-(7).

Step 5: judge whether the vulnerability index calculation of all key nodes and key sections is complete. If so, go to Step 6; otherwise, return to Step 4.

Step 6: terminate the algorithm and output the ranking results of vulnerability indicators.

\section{Application}

4.1. Data Preparation. In this study, the road network of the Nanshan Science and Technology Park area in Shenzhen City, Guangdong Province, China, was used to verify the above research methods, models, and algorithms. The Science and Technology Park was funded and constructed in 2001 and covers an area of 706,000 square meters and a construction area of 3 million square meters. The park was in an advantageous geographical environment, rich cultural atmosphere, and numerous scientific research institutes. The road traffic network is an important infrastructure to support the social and economic development of the area.

According to statistics, at present, there are approximately 450,000 people employed in the Science and Technology Park area in Nanshan District of Shenzhen City, with a construction area of about 11 million square meters. It is estimated that by 2025, it will reach 800,000 people and 22.5 million square meters, and the existing road carrying capacity can only meet approximately $71 \%$ of the current travel demand. At present the average speed of the road network in the area is lower than the international congestion warning line $(20 \mathrm{~km}$ per hour). According to the calculation, the traffic capacity of the main roads (approximately 28,000 


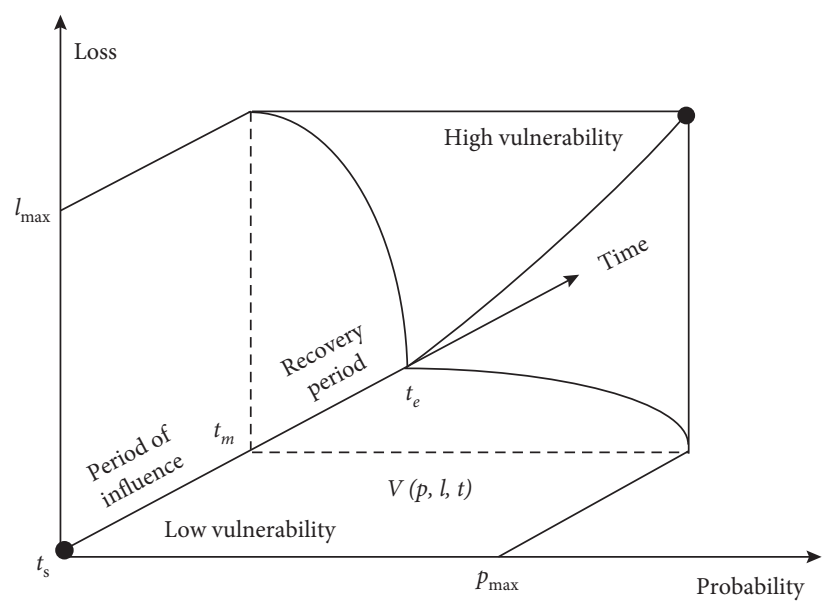

FigURE 1: Three-dimensional model of road network vulnerability concept.

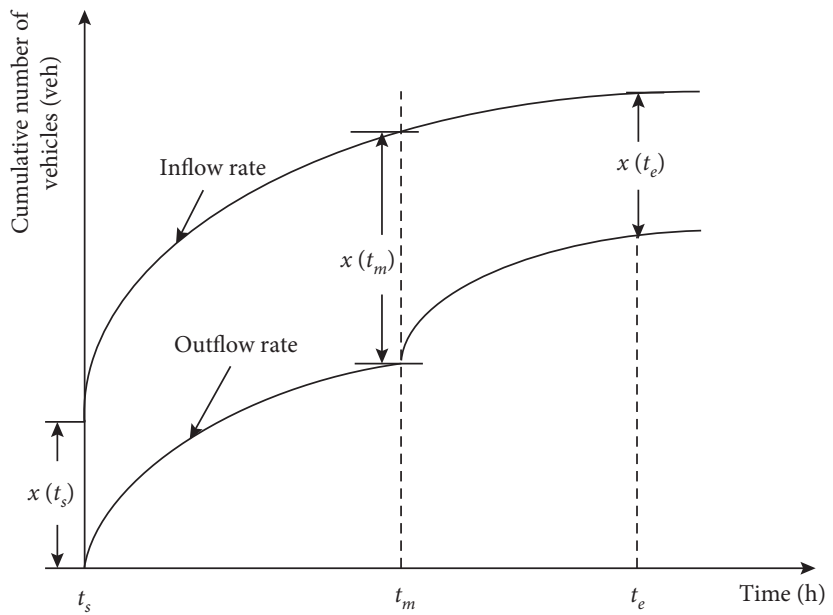

Figure 2: Schematic diagram of road traffic volume change after emergency.

vehicles/peak hour) cannot meet the demand of vehicles (approximately 40,000//peak hour). Figure 3 shows the electronic map of the local road network in this area as extracted by ArcGIS software. According to the research scheme, the backbone road network was selected on the electronic map, and the corresponding road network topology was constructed using the vision software. Figure 4 shows that there are 35 nodes and 59 arc segments, including two starting points (origin points) and two ending points (destination points). The arc segment of a single arrow indicated that the road section was one-way traffic, and the arrow at both ends indicates that the road section was twoway traffic. Based on the data provided by the department in charge of urban road traffic, the research group obtained the road traffic data of the area in combination with the field investigation (see Table 2).

4.2. Emergency Scenario and Impact Analysis. Emergency mainly refers to natural and accidental disasters, public health, and social security events that suddenly occur or may cause serious social harm where emergency measures are required to deal with them. These events were characterized by strong randomness, wide coverage, and significant negative diffusion effect. When an emergency occurs, on the one hand, it leads to interruption and changes the topological structure of the relevant road network; on the other hand, due to these changes, traffic and transportation activities are affected, and the travel demand also changes accordingly. With different degrees of information suspension and different audiences, the impact of emergency on road traffic activities would be different.

According to the stated preference (SP) survey, when the accident occurs, the road users in the regional road network could be divided into two types: (1) those who wait for the road section to be repaired, reopen, and drive according to their original road plan after receiving the information; (2) those who to return to the nearest exit through the reverse evacuation of traffic to find the shortest alternative path. Taking the topological road network shown in Figure 4 as example, it was assumed that there was a serious traffic accident on section 37 of the road network, which may produce the following two effects: (1) once the accident occurred, section 37 was interrupted. The vehicles originally 
passing through section 37 would be diverted to other nearby roads, which would exert pressure on related traffic, and the traffic in this area would be redistributed; (2) after the accident, intersection 25 and 13 would be blocked. Due to the interruption of section 37, the traffic flow of two related intersections was blocked, and the intersection was affected by vehicle queuing, resulting in cascading failure. As a consequence, the topological structure of the road network would change to some extent.

4.3. Investigation of Road Users' Travel Choice Behavior. To obtain the characteristic parameters of road users' travel choice behavior under the emergency conditions and to obtain the probability of the same after the accident and its impact on the road traffic operation state, we investigated road users' travel choice behavior based on the set emergency scenario. The survey's purpose was to identify how those who originally planned to use the road network would adapt and select an alternative path once the original planned path had been interrupted. The possible situations include three travel plan choices: cancel travel, suspend travel, and continue with normal travel. In terms of travel mode selection, let us assume that there were three modes of transportation: private car, bus, and taxi. The survey content mainly included the influence of personal attributes (mainly occupation and age) and the travel purpose of road users, and the travel time and travel mode choice behavior under different travel purposes under the characteristics of road users' personal information and emergency.

Based on the survey, this project designed an SP questionnaire. Through the combination of online and offline surveys, 218 valid samples were finally recovered, which met the minimum requirements of statistical survey samples (157), and reflected the travel choices of different individuals in emergency situations. To quantitatively analyze the impact of personal attributes and options on travel results, this project used the stochastic utility logit model to establish the probability model of travel time and travel mode selection.

4.3.1. Determination of the Utility Function and Parameter Estimation. The previous discussion shows that if $N$ was used to represent the set of possible travel options, and $I$ was used to represent the set of traveler types, then the random utility $U_{n}^{i}$ of type $i$ travelers choosing the $n$ travel option could be expressed as follows:

$$
U_{n}^{i}=V_{n}^{i}+\varepsilon_{n}^{i}, \quad n \in N, i \in I,
$$

where $V_{n}^{i}$ was the determinable utility of type $i$ travelers for the $n$ travel scheme, $\varepsilon_{n}^{i}$ was the random error term, and both obeyed the Gumbel distribution. According to the multinomial logit (ML) model, the number of samples is set as $I$, and $\delta_{i n}$ as the probability variable. The probability of simultaneous implementation of each option was as follows:

$$
p_{1 n}^{\delta_{1 n}}, p_{2 n}^{\delta_{2 n}} \ldots p_{i n}^{\delta_{i n}} \ldots p_{I n}^{\delta_{I n}}=\prod_{i \in I} p_{i n}^{\delta_{i n}}
$$

So, the simultaneous probability $L^{*}$ of travelers was as follows:

$$
L^{*}=\prod_{n=1}^{N} \prod_{i \in I} p_{i n}^{\delta_{i n}} .
$$

Equation (22) represents the likelihood function of $\mathrm{ml}$. According to equation (13), the log likelihood function could be obtained as follows:

$$
L=\ln L^{*}=\sum_{n=1}^{N} \sum_{i \in I} \delta_{i n} \ln p_{i n}=\sum_{n=1}^{N} \sum_{i \in I} \delta_{i n}\left(\theta X_{i n}-\ln \sum_{j \in I} e^{\theta X_{j n}}\right) .
$$

In the above equation, $X$ was the characteristic variable of road users' travel choice, $\theta$ was the parameter to be estimated corresponding to the characteristic variable, and $L$ was a convex function about $\theta$, and using $\theta$ to derive the two sides of the equation and setting the value to 0 , the maximum likelihood estimation value $\hat{\theta}$ of $\theta$ could be obtained. Here, the parameters of the model were estimated using SPSS software.

\subsubsection{Parameter Calculation and Result Analysis. Taking} cancellation, postponement, private car, bus, and taxi as travel options, through the correlation analysis of the survey data, the characteristics of the options and that of the travelers were finally determined (see Table 3 ). The values of the characteristic variables were obtained through actual investigation.

The logistic module of the SPSS software was used to estimate the model parameters. The model characteristic variables corresponding to the normal travel time selection and the parameter estimation values of the corresponding characteristic variables of other transportation modes with the bus as the travel reference in the travel mode selection were obtained. Tables 4 and 5 show the calculation results.

According to the principle of statistics, when the degree of freedom is 1 and the confidence is 0.05 , the critical value of the Wald parameter test is 3.841 . The larger the Wald test value, the more significant the correlation between independent and dependent variables. From the above Wald test value, we observed that the selected characteristic variables were the factors that had a significant impact on road users' travel choice behavior. Simultaneously, travel time, vehicle ownership, and age had a significant impact on road users' travel mode choice. Table 6 shows the test results for the overall likelihood ratio test of the model.

The confidence interval of the model parameter estimation was 95\%, which is shown in Table 6 from the likelihood ratio test results, which demonstrated that the model had significant importance. According to the conditions of superior ratio, the fitting effect of the model was good. For the whole model, the value of MC Fadden's coefficient $p^{2}$ was between 0.2 and 0.4 , which demonstrates good accuracy. Table 7 shows the calibration results of the traveler's nonintegrator parameters. 
TABLE 1: Vulnerability scale of key nodes or road sections.

\begin{tabular}{lccccc}
\hline & \multicolumn{5}{c}{ Vulnerability level } \\
& 1 & 2 & 3 & 4 & 5 \\
\hline Grade scale & {$[0,0.51]$} & {$[0.51,0.63]$} & {$[0.63,0.80]$} & {$[0.80,0.91]$} & {$[0.91,1]$} \\
Vulnerability & Very low & Low & Medium & High & Very high \\
\hline
\end{tabular}

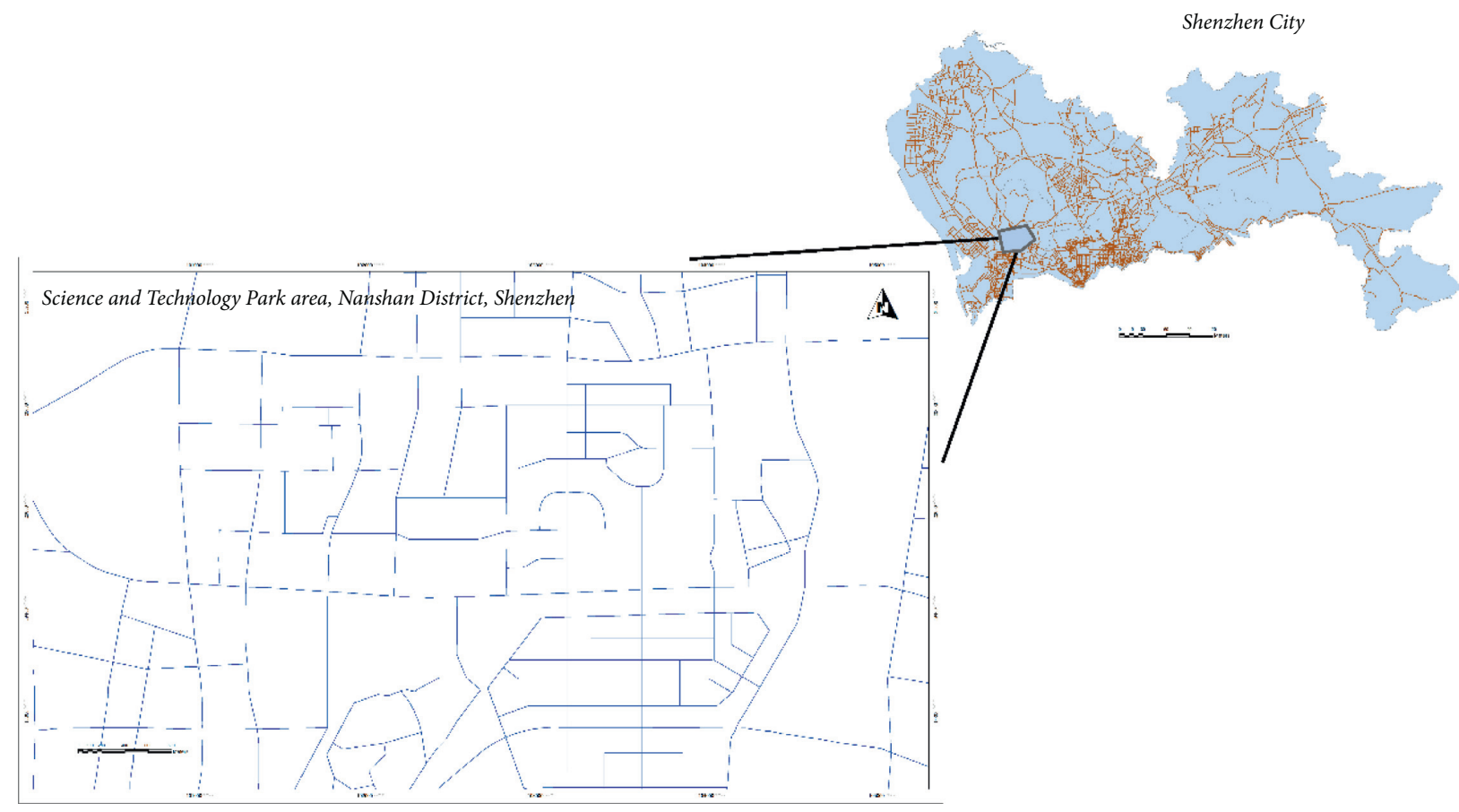

Figure 3: Electronic map of road network.

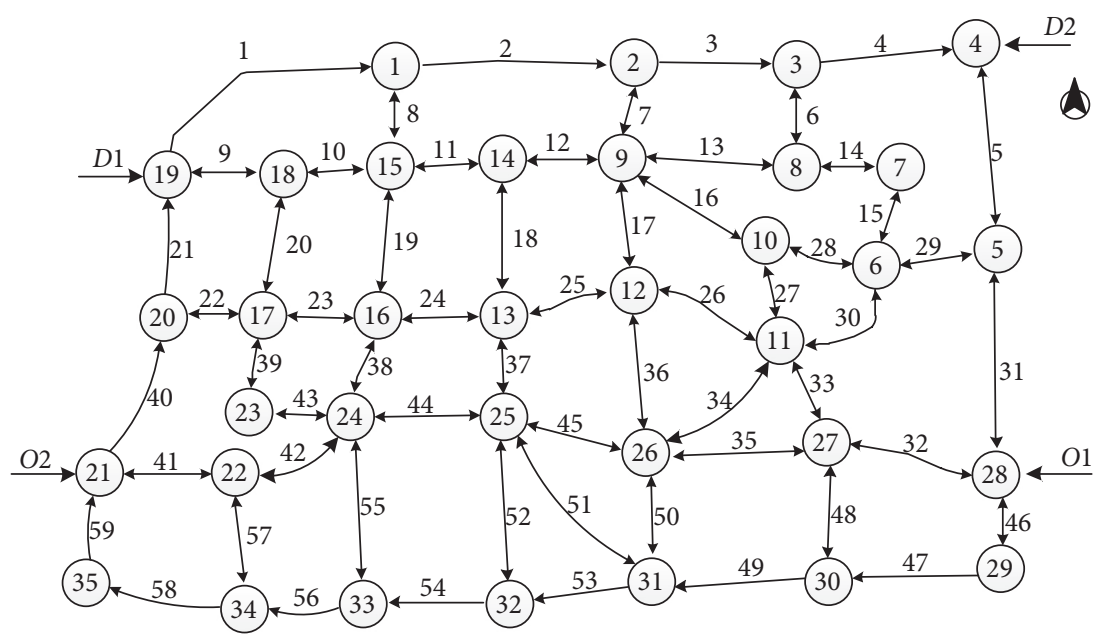

FigURE 4: Backbone road network topology. 
TABLE 2: Survey results of road traffic network data.

\begin{tabular}{|c|c|c|c|c|c|c|c|}
\hline Nodes (r) & Nodes (s) & $\operatorname{Arcs}(a)$ & Lane & Length (m) & $\begin{array}{c}\text { Designing } \\
\text { velocity }(\mathrm{km} / \mathrm{h})\end{array}$ & $\begin{array}{l}\text { Free travelling } \\
\text { time }(\mathrm{h})\end{array}$ & $\begin{array}{c}\text { Designing } \\
\text { capacity }(\mathrm{pcu} / \mathrm{h})\end{array}$ \\
\hline 19 & 1 & 1 & 4 & 0.697 & 60 & 0.0116 & 3100 \\
\hline 1 & 2 & 2 & 4 & 0.609 & 60 & 0.0102 & 3100 \\
\hline 2 & 3 & 3 & 3 & 0.386 & 60 & 0.0064 & 2325 \\
\hline 3 & 4 & 4 & 3 & 0.454 & 60 & 0.0076 & 2325 \\
\hline 4 & 5 & 5 & 4 & 0.554 & 60 & 0.0092 & 3100 \\
\hline 5 & 4 & 5 & 4 & 0.554 & 60 & 0.0092 & 3100 \\
\hline 3 & 8 & 6 & 4 & 0.212 & 40 & 0.0053 & 2900 \\
\hline 8 & 3 & 6 & 4 & 0.212 & 40 & 0.0053 & 2900 \\
\hline 2 & 9 & 7 & 4 & 0.238 & 60 & 0.004 & 3100 \\
\hline 9 & 2 & 7 & 4 & 0.238 & 60 & 0.004 & 3100 \\
\hline 1 & 15 & 8 & 4 & 0.256 & 50 & 0.0051 & 3000 \\
\hline 15 & 1 & 8 & 4 & 0.256 & 50 & 0.0051 & 3000 \\
\hline 18 & 19 & 9 & 6 & 0.32 & 60 & 0.0053 & 4650 \\
\hline 19 & 18 & 9 & 6 & 0.32 & 60 & 0.0053 & 4650 \\
\hline 18 & 15 & 10 & 6 & 0.258 & 60 & 0.0043 & 4650 \\
\hline 15 & 18 & 10 & 6 & 0.258 & 60 & 0.0043 & 4650 \\
\hline 15 & 14 & 11 & 6 & 0.258 & 60 & 0.0043 & 4650 \\
\hline 14 & 15 & 11 & 6 & 0.258 & 60 & 0.0043 & 4650 \\
\hline 9 & 14 & 12 & 6 & 0.353 & 60 & 0.0059 & 4650 \\
\hline 14 & 9 & 12 & 6 & 0.353 & 60 & 0.0059 & 4650 \\
\hline 8 & 9 & 13 & 6 & 0.389 & 60 & 0.0065 & 4650 \\
\hline 9 & 8 & 13 & 6 & 0.389 & 60 & 0.0065 & 4650 \\
\hline 7 & 8 & 14 & 4 & 0.566 & 50 & 0.0113 & 3000 \\
\hline 8 & 7 & 14 & 4 & 0.566 & 50 & 0.0113 & 3000 \\
\hline 6 & 7 & 15 & 6 & 0.259 & 40 & 0.0065 & 4400 \\
\hline 7 & 6 & 15 & 6 & 0.259 & 40 & 0.0065 & 4400 \\
\hline 9 & 10 & 16 & 4 & 0.269 & 40 & 0.0067 & 2900 \\
\hline 10 & 9 & 16 & 4 & 0.269 & 40 & 0.0067 & 2900 \\
\hline 9 & 12 & 17 & 6 & 0.325 & 60 & 0.0054 & 4650 \\
\hline 12 & 9 & 17 & 6 & 0.325 & 60 & 0.0054 & 4650 \\
\hline 13 & 14 & 18 & 6 & 0.373 & 60 & 0.0062 & 4650 \\
\hline 14 & 13 & 18 & 6 & 0.373 & 60 & 0.0062 & 4650 \\
\hline 15 & 16 & 19 & 6 & 0.379 & 60 & 0.0063 & 4650 \\
\hline 16 & 15 & 19 & 6 & 0.379 & 60 & 0.0063 & 4650 \\
\hline 17 & 18 & 20 & 4 & 0.383 & 60 & 0.0064 & 3100 \\
\hline 18 & 17 & 20 & 4 & 0.383 & 60 & 0.0064 & 3100 \\
\hline 20 & 19 & 21 & 6 & 0.38 & 60 & 0.0063 & 4650 \\
\hline 20 & 17 & 22 & 6 & 0.249 & 40 & 0.0062 & 4400 \\
\hline 17 & 20 & 22 & 6 & 0.249 & 40 & 0.0062 & 4400 \\
\hline 16 & 17 & 23 & 6 & 0.271 & 40 & 0.0068 & 4400 \\
\hline 17 & 16 & 23 & 6 & 0.271 & 40 & 0.0068 & 4400 \\
\hline 13 & 16 & 24 & 6 & 0.335 & 40 & 0.0084 & 4400 \\
\hline 16 & 13 & 24 & 6 & 0.335 & 40 & 0.0084 & 4400 \\
\hline 12 & 13 & 25 & 6 & 0.359 & 40 & 0.009 & 4400 \\
\hline 13 & 12 & 25 & 6 & 0.359 & 40 & 0.009 & 4400 \\
\hline 11 & 12 & 26 & 6 & 0.49 & 40 & 0.0123 & 4400 \\
\hline 12 & 11 & 26 & 6 & 0.49 & 40 & 0.0123 & 4400 \\
\hline 10 & 11 & 27 & 4 & 0.535 & 50 & 0.0107 & 3000 \\
\hline 11 & 10 & 27 & 4 & 0.535 & 50 & 0.0107 & 3000 \\
\hline 6 & 10 & 28 & 4 & 0.486 & 40 & 0.0122 & 2900 \\
\hline 10 & 6 & 28 & 4 & 0.486 & 40 & 0.0122 & 2900 \\
\hline 5 & 6 & 29 & 6 & 0.296 & 60 & 0.0049 & 4650 \\
\hline 6 & 5 & 29 & 6 & 0.296 & 60 & 0.0049 & 4650 \\
\hline 6 & 11 & 30 & 4 & 0.302 & 40 & 0.0076 & 2930 \\
\hline 11 & 6 & 30 & 4 & 0.302 & 40 & 0.0076 & 2930 \\
\hline 5 & 28 & 31 & 6 & 0.535 & 60 & 0.0089 & 4650 \\
\hline 28 & 5 & 31 & 6 & 0.535 & 60 & 0.0089 & 4650 \\
\hline 27 & 28 & 32 & 4 & 0.435 & 60 & 0.0073 & 3100 \\
\hline 28 & 27 & 32 & 4 & 0.435 & 60 & 0.0073 & 3100 \\
\hline 11 & 27 & 33 & 4 & 0.254 & 50 & 0.0051 & 3000 \\
\hline 27 & 11 & 33 & 4 & 0.254 & 50 & 0.0051 & 3000 \\
\hline 11 & 26 & 34 & 6 & 0.537 & 40 & 0.0134 & 4400 \\
\hline
\end{tabular}


TABle 2: Continued.

\begin{tabular}{|c|c|c|c|c|c|c|c|}
\hline Nodes (r) & Nodes (s) & $\operatorname{Arcs}(\mathrm{a})$ & Lane & Length $(\mathrm{m})$ & $\begin{array}{c}\text { Designing } \\
\text { velocity }(\mathrm{km} / \mathrm{h})\end{array}$ & $\begin{array}{c}\text { Free travelling } \\
\text { time }(\mathrm{h})\end{array}$ & $\begin{array}{c}\text { Designing } \\
\text { capacity }(\mathrm{pcu} / \mathrm{h})\end{array}$ \\
\hline 26 & 11 & 34 & 6 & 0.537 & 40 & 0.0134 & 4400 \\
\hline 26 & 27 & 35 & 6 & 0.441 & 60 & 0.0074 & 4650 \\
\hline 27 & 26 & 35 & 6 & 0.441 & 60 & 0.0074 & 4650 \\
\hline 12 & 26 & 36 & 6 & 0.422 & 60 & 0.007 & 4650 \\
\hline 26 & 12 & 36 & 6 & 0.422 & 60 & 0.007 & 4650 \\
\hline 13 & 25 & 37 & 6 & 0.243 & 60 & 0.0041 & 4650 \\
\hline 25 & 13 & 37 & 6 & 0.243 & 60 & 0.0041 & 4650 \\
\hline 16 & 24 & 38 & 6 & 0.428 & 60 & 0.0071 & 4650 \\
\hline 24 & 16 & 38 & 6 & 0.428 & 60 & 0.0071 & 4650 \\
\hline 17 & 23 & 39 & 6 & 0.393 & 60 & 0.0066 & 4650 \\
\hline 23 & 17 & 39 & 6 & 0.393 & 60 & 0.0066 & 4650 \\
\hline 20 & 21 & 40 & 6 & 0.42 & 60 & 0.007 & 4650 \\
\hline 21 & 20 & 40 & 6 & 0.42 & 60 & 0.007 & 4650 \\
\hline 21 & 22 & 41 & 6 & 0.355 & 60 & 0.0059 & 4650 \\
\hline 22 & 21 & 41 & 6 & 0.355 & 60 & 0.0059 & 4650 \\
\hline 22 & 24 & 42 & 6 & 0.361 & 60 & 0.006 & 4650 \\
\hline 24 & 22 & 42 & 6 & 0.361 & 60 & 0.006 & 4650 \\
\hline 23 & 24 & 43 & 6 & 0.278 & 40 & 0.007 & 4400 \\
\hline 24 & 23 & 43 & 6 & 0.278 & 40 & 0.007 & 4400 \\
\hline 24 & 25 & 44 & 6 & 0.387 & 40 & 0.0097 & 4400 \\
\hline 25 & 24 & 44 & 6 & 0.387 & 40 & 0.0097 & 4400 \\
\hline 25 & 26 & 45 & 6 & 0.473 & 50 & 0.0095 & 4500 \\
\hline 26 & 25 & 45 & 6 & 0.473 & 50 & 0.0095 & 4500 \\
\hline 28 & 29 & 46 & 6 & 0.264 & 60 & 0.0044 & 4650 \\
\hline 29 & 28 & 46 & 6 & 0.264 & 60 & 0.0044 & 4650 \\
\hline 29 & 30 & 47 & 8 & 0.419 & 60 & 0.007 & 6200 \\
\hline 27 & 30 & 48 & 6 & 0.344 & 60 & 0.0057 & 4650 \\
\hline 30 & 27 & 48 & 6 & 0.344 & 60 & 0.0057 & 4650 \\
\hline 30 & 31 & 49 & 8 & 0.444 & 60 & 0.0074 & 6200 \\
\hline 26 & 31 & 50 & 6 & 0.351 & 60 & 0.0059 & 4650 \\
\hline 31 & 26 & 50 & 6 & 0.351 & 60 & 0.0059 & 4650 \\
\hline 25 & 31 & 51 & 6 & 0.588 & 50 & 0.0118 & 4500 \\
\hline 31 & 25 & 51 & 6 & 0.588 & 50 & 0.0118 & 4500 \\
\hline 25 & 32 & 52 & 6 & 0.351 & 60 & 0.0059 & 4650 \\
\hline 32 & 25 & 52 & 6 & 0.351 & 60 & 0.0059 & 4650 \\
\hline 31 & 32 & 53 & 8 & 0.36 & 60 & 0.006 & 6200 \\
\hline 32 & 33 & 54 & 8 & 0.38 & 60 & 0.0063 & 6200 \\
\hline 24 & 33 & 55 & 6 & 0.463 & 60 & 0.0077 & 4650 \\
\hline 33 & 24 & 55 & 6 & 0.463 & 60 & 0.0077 & 4650 \\
\hline 33 & 34 & 56 & 8 & 0.291 & 60 & 0.0049 & 6200 \\
\hline 22 & 34 & 57 & 6 & 0.329 & 60 & 0.0055 & 4650 \\
\hline 34 & 22 & 57 & 6 & 0.329 & 60 & 0.0055 & 4650 \\
\hline 34 & 35 & 58 & 8 & 0.368 & 60 & 0.0061 & 6200 \\
\hline 35 & 21 & 59 & 6 & 0.283 & 60 & 0.0047 & 4650 \\
\hline
\end{tabular}

According to the calculation results of variable parameters, the probability calculation model of travel time selection under the sudden road fuel leakage and major traffic accidents can be determined, and the expression is as follows:

$$
\ln \left(p_{i j}\right)=\sum_{k=1}^{6} \theta_{k j} X_{i k j}, \quad j=1,2,3,4,5,
$$

where $\sum_{j=1}^{5} P_{i j}=1, P_{i 1} \sim P_{i 5}$ represents the probability of cancelling, delaying, and travelling by private car, bus, and taxi for type $i$ travelers, and $\theta_{k j}$ represents the estimated parameters corresponding to different characteristic variables under travel choice.

4.4. Calculation of OD Traffic Generation in Residential Area. According to the travel time and travel mode selection probabilities obtained above, the traffic generation of a certain type of traffic mode in a certain period can be determined in the second district under an emergency, based on the personal classification method in the calculation of total traffic generation, as follows: 
TABLE 3: Selection of model characteristic variables.

\begin{tabular}{|c|c|c|c|c|c|c|c|c|c|c|}
\hline \multirow{3}{*}{ Utility } & \multicolumn{5}{|c|}{$\begin{array}{c}\text { Selection of } \\
\text { characteristic variables }\end{array}$} & \multirow{2}{*}{\multicolumn{5}{|c|}{ Traveler characteristic variables }} \\
\hline & \multicolumn{4}{|c|}{$\begin{array}{l}\text { Intrinsic dumb } \\
\text { element }\end{array}$} & \multirow{2}{*}{$\begin{array}{c}\text { Common } \\
\text { variable } \\
\text { Travel time } \\
\left(X_{i n 5}\right)\end{array}$} & & & & & \\
\hline & $X_{i n 1}$ & $X_{i n 2}$ & $X_{i n 3}$ & $X_{i n 4}$ & & Gender $\left(X_{i n 6}\right)$ & Age $\left(X_{i n 7}\right)$ & Occupation $\left(X_{i n 8}\right)$ & $\begin{array}{c}\text { Vehicle } \\
\text { ownership }\left(X_{i n 9}\right)\end{array}$ & $\begin{array}{c}\text { Travel } \\
\text { characteristics }\left(X_{i n 10}\right)\end{array}$ \\
\hline $\begin{array}{l}\text { Cancel } \\
\left(V_{1 n}\right)\end{array}$ & 1 & 0 & 0 & 0 & $X_{1 n 5}$ & $X_{1 n 6}$ & $X_{1 n 7}$ & $X_{1 n 8}$ & $X_{1 n 9}$ & $X_{1 n 10}$ \\
\hline $\operatorname{Delay}\left(V_{2 n}\right)$ & 0 & 1 & 0 & 0 & $X_{2 n 5}$ & $X_{2 n 6}$ & $X_{2 n 7}$ & $X_{2 n 8}$ & $X_{2 n 9}$ & $X_{2 n 10}$ \\
\hline $\begin{array}{l}\text { Private car } \\
\left(V_{3 n}\right)\end{array}$ & 0 & 0 & 1 & 0 & $X_{3 n 5}$ & $X_{3 n 6}$ & $X_{3 n 7}$ & $X_{3 n 8}$ & $X_{3 n 9}$ & $X_{3 n 10}$ \\
\hline Bus $\left(V_{4 n}\right)$ & 0 & 0 & 0 & 1 & $X_{4 n 5}$ & 0 & 0 & 0 & 0 & $X_{4 n 10}$ \\
\hline $\operatorname{Taxi}\left(V_{5 n}\right)$ & 0 & 0 & 0 & 0 & $X_{5 n 5}$ & 0 & 0 & 0 & 0 & $X_{5 n 10}$ \\
\hline $\begin{array}{l}\text { Pending } \\
\text { parameters }\end{array}$ & $\theta_{1}$ & $\theta_{2}$ & $\theta_{3}$ & $\theta_{4}$ & $\theta_{5}$ & $\theta_{6}$ & $\theta_{7}$ & $\theta_{8}$ & $\theta_{9}$ & $\theta_{10}$ \\
\hline
\end{tabular}

TABLE 4: Estimation results of characteristic variable parameters of the travel time choice model.

\begin{tabular}{lcccc}
\hline & \multicolumn{2}{c}{ Cancel travel } & \multicolumn{2}{c}{ Delay travel } \\
& Parameter & Wald & Parameter & Wald \\
\hline Travel time & -0.17 & 4.83 & -0.21 & 3.96 \\
Gender & 0.65 & 3.81 & 0.42 & 2.01 \\
Age & 0.53 & 2.01 & 0.25 & 1.86 \\
Occupation & -0.08 & 3.82 & -0.17 & 3.92 \\
Vehicle ownership & 0.34 & 5.45 & 2.81 & 5.33 \\
Travel characteristics & 3.11 & 2.45 & 2.34 & 4.09 \\
\hline
\end{tabular}

TABLE 5: Estimation results of characteristic variable parameters of the travel mode selection model.

\begin{tabular}{lcccc}
\hline & \multicolumn{2}{c}{ Private car } & Taxi & Parameter \\
& Parameter & Wald & 4.53 & 0.19 \\
Travel time & 0.21 & 1.87 & -0.72 & 0.08 \\
Gender & -0.06 & 2.11 & -0.64 & 3.85 \\
Age & 0.22 & 3.93 & 0.07 & 4.76 \\
Occupation & 0.37 & 5.95 & 3.21 & 2.11 \\
Vehicle ownership & 0.42 & 4.28 & 3.45 & 0.32 \\
Travel characteristics & 0.32 & & 0.42 \\
\hline
\end{tabular}

TABLE 6: Model likelihood ratio test.

\begin{tabular}{lcccr}
\hline & $X^{2}$ & $d_{f}$ & Significant level (sig.) & $\rho^{2}$ \\
\hline Travel time selection & 89.154 & 29 & 0.000 & 0.351 \\
Choice of travel mode & 81.326 & 28 & 0.000 & 0.296 \\
\hline
\end{tabular}

$$
q_{r n}=N_{r} \sum_{i} a_{i r} S_{i} p_{i n}, \quad i \in I
$$

where $q_{r n}$ was the traffic generating capacity of all types of road users in community $r$ choosing travel mode $n, N_{r}$ was the total population of all types of road users in community $r, a_{i r}$ was the proportion of class $i$ road users in community $r$, $S_{i}$ was the average number of trips per person of class $i$ road users in a certain period of time, and $p_{\text {in }}$ was the probability of class $i$ road users choosing travel mode.
4.5. Traffic Vulnerability Identification of Road Network. According to the design of the vulnerability comprehensive identification index, the traffic VURN under emergency situations could be identified by the comprehensive measurement index of the traffic VURN, and the comprehensive measurement index was obtained by a weighted synthesis of three types of indexes, which were road network connectivity and its change index under emergency conditions and road network vulnerability under the same, network efficiency and its change index, and network traffic 
TABLE 7: Traveler type and nonaggregate parameter calibration.

\begin{tabular}{lcccc}
\hline Travel choice & Proportion $\left(u_{d}^{r s}\right)$ & Parameter $(\theta)$ & Constant $\left(\theta_{0}\right)$ & Selection probability $\left(P_{\text {in }}\right)$ \\
\hline Cancel & 0.245 & 1.15 & 0.14 & 0.72 \\
Suspend & 0.376 & 1.52 & 0.09 & 0.88 \\
Private car & 0.151 & 0.94 & 0.12 & 0.61 \\
Bus & 0.211 & 1.623 & 0.23 & 0.89 \\
Taxi & 0.017 & 0.264 & 0.04 & 0.45 \\
\hline
\end{tabular}

performance and its change index in an emergency. Therefore, it was necessary to calculate three types of indicators and subsequently synthesize these to obtain the final vulnerability identification results.

\subsection{Road Network Connectivity Changes during Emergency.} We constructed the node adjacency matrix of the road network in the Nanshan Science and Technology Park area of Shenzhen City, analyzed the topological characteristics before and after the emergency using the connectivity or network efficiency index, and calculated the changes of the connectivity or network efficiency index of the point or edge before and after the emergency according to equations (1) and (2). Prior to emergency, there were 35 nodes and 59 road sections in the road network, and the connectivity $G=0.596$. After emergency, after each road node was interrupted one by one, the connectivity $G^{\prime}$ of the road network after the accident was calculated, and the statistical results are shown in Figure 5. Figure 5 shows the changes of network connectivity of road network nodes before and after the emergency. The nodes with $\Delta G>0.03$ were nodes $9,11,24$, 25 , and 26 . Relatively speaking, these nodes were vulnerable and required supervision. Table 8 shows the corresponding road sections.

\subsection{Road Network Efficiency Changes during Emergency.} The change of road network efficiency under emergency could also reflect the vulnerability of the road network from one side. According to the previous assumption, we considered that each road section in Figure 4 had a major accident, which led to the interruption of the road section and the traffic efficiency changed. Then, we calculated the traffic efficiency $E$ after the accident according to equations (3) and (4). To calculate road network traffic efficiency, it was necessary to obtain the shortest path between OD pairs. Here, the Dijkstra algorithm was used for this purpose.

In Figure 6, the calculation shows that the network efficiency of the topology was 0.6742 prior to emergency. After the nodes were interrupted, the efficiency of the road network was $E^{\prime}$. By comparing the efficiency before and after the emergency, the change $\Delta E$ was obtained, and Figure 6 shows the calculation results statistically.

Figure 6 shows a three-dimensional statistical chart composed of road sections (arcs), traffic efficiency vulnerability index $\left(E^{\prime}\right)$ after the accident, and traffic efficiency vulnerability index $(\Delta E)$ before and after the accident. The list on the right side shows some statistical ranking results of link vulnerability. Figure 6 shows that section 42 had the greatest impact on traffic efficiency on the whole road network, played an important role, and was the most vulnerable section. Except for section 42, sections 37, 30, 10, and 16 with the change of road network efficiency more than 0.015 were arranged according to the influence of traffic efficiency from large to small. These sections played an important role in the stability of the road network and required high attention in daily maintenance.

\subsection{Road Network Traffic Performance Changes during} Emergency. The investigation showed that emergency could easily lead to urban road damage and traffic interruption. Among them, road damage would lead to changes in the structure of the road network, which would affect the connectivity and traffic efficiency. In addition, the accident would impact the traffic operation of the road unit, such that the road transportation function would be affected. In this case, for travelers, according to the amount of information they receive, they would choose to cancel, suspend, or travel through other modes of transportation, and the on-the-way traffic would find alternative routes, which would lead to the redistribution of urban road network traffic. For a certain period, the corresponding road sections would frequently develop long queues. Figure 2 shows the overall operation characteristics of network traffic and the distribution characteristics of section traffic flow. To analyze the vulnerability based on the traffic flow operation model, the following assumptions for the construction of the model were made:

(1) Suppose the queue was at the vehicle point, and the length of each vehicle is 0

(2) The queuing capacity of each road section in the network was limited, which could not hold an infinite number of vehicles in line

(3) The section capacity would rapidly decrease to 0 under the condition of sudden congestion

(4) If the same lane ran in a single row, and there was no multivehicle parallel situation, the traffic capacity of the congested road section would be approximately equal to the maximum queuing capacity when congestion ceased

According to the road network distribution in Figure 4 and the survey data in Table 2, three typical OD pairs were selected to load. Using MATLAB, the Dijkstra algorithm was used to calculate the corresponding shortest path of each OD pair under normal conditions. Then, according to the set emergency, the above algorithm was used to interrupt the road operation after each incident, and the traffic 


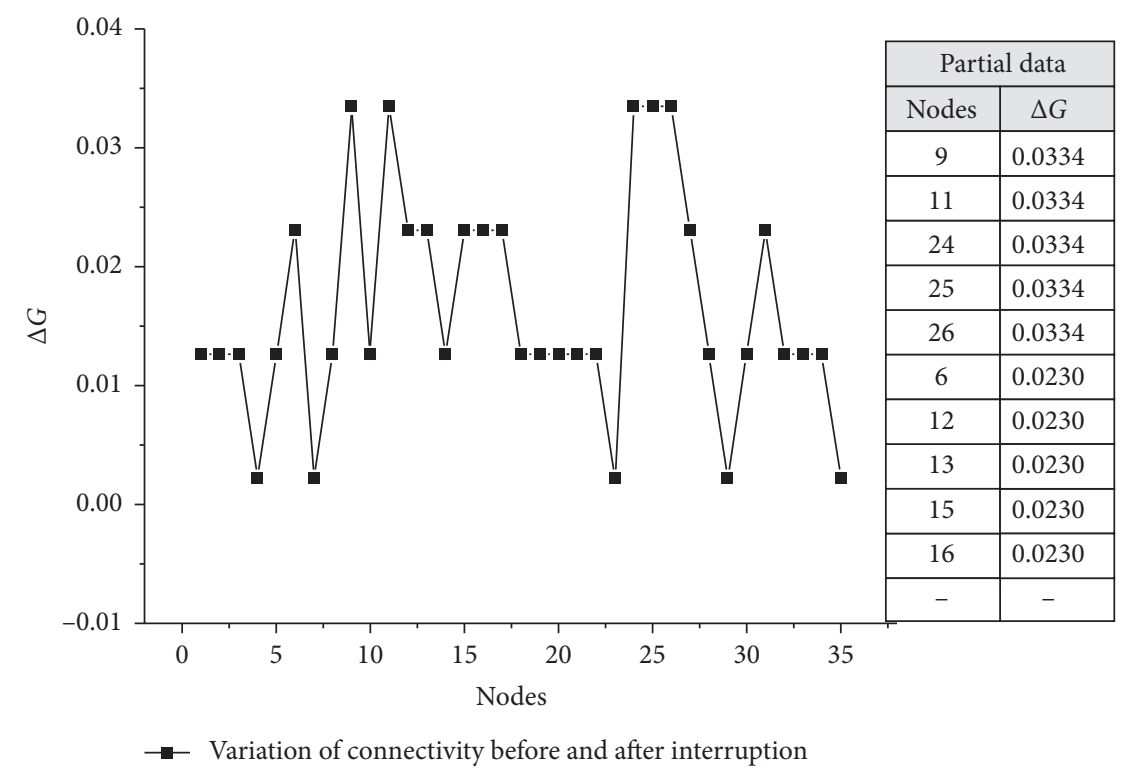

Figure 5: Changes of the connectivity of network before and after emergency.

distribution results after traffic allocation, after emergency, were obtained. This involved equations (5) through (7) and the algorithm designed earlier in this paper. In the calculation process, parameters $\alpha$ and $\beta$ of the BPR function were 0.15 and 4, respectively. Finally, the change values of all traffic performance parameters were obtained, Table 8 shows the statistics.

Table 8 shows that after emergency, the interruption caused traffic demand change, which led to the redistribution of road traffic to a new equilibrium state, resulting in travel time loss. Among them, the total travel loss time of sections $23,5,25,12$, and 44 after interruption was more than $31.2 \mathrm{~min}$. This was the key section of the whole road network, which in turn required significant attention from the relevant department. Figure 7 shows the convergence of the vulnerability comprehensive identification model and the algorithm. The iterative gap value decreased rapidly with the increase of the iteration time. After 10 iterations, the satisfactory equilibrium numerical solution was achieved, which demonstrated that the model solution algorithm proposed was highly efficient.

\section{Analysis}

In the previous part of network vulnerability identification, we analyze the topological connectivity, traffic efficiency, and performance of the network during emergency. The comprehensive identification of traffic VURN was analyzed from two perspectives. First, the traffic vulnerability of the road network was identified by considering both topological connectivity and network efficiency. Second, the traffic vulnerability was identified by considering topological connectivity, network efficiency, and traffic performance. On this basis, the results of road network vulnerability identification from two different perspectives were compared and analyzed.

5.1. Analysis of Vulnerability Identification Results considering Network Connectivity and Efficiency. The results calculated in the previous section were counted in descending order, including the change value of road network connectivity $(\Delta G)$, and that of road network efficiency $(\Delta E)$ and the corresponding nodes and sections. Table 9 only lists the first 10 rows of the results due to spacing issues. From these results, we observed that the top 10 nodes were nodes 9,11 , $24,25,26,6,12,13,15$, and 16, which indicated that the vulnerability of these nodes had changed from strong to weak. According to the correlation between nodes and connected edges, when these nodes were impacted by emergency, their corresponding connected edges were also affected. Therefore, the corresponding connected edges of these nodes would also show the corresponding vulnerability. Based on this, the connected edges of these nodes were statistically analyzed.

The connection edges corresponding to the above nodes had a strong correlation with the connection edges after the statistics of the change value of network efficiency $(\Delta E)$. In other words, the nodes corresponding to the connection edges obtained by sorting the change value of network efficiency $(\Delta E)$ from large to small were highly consistent with the nodes arranged by the change value of the network connectivity index $(\Delta G)$ from large to small. From the top 10 nodes listed, there were seven nodes in total, including 9,11 , $24,25,6,13$, and 15, which coincided with the nodes corresponding to the network efficiency connecting edge. This indicated that prior to and after emergency, the change value of network connectivity $(\Delta G)$ and that of network efficiency 
TABLE 8: Calculation results of traffic performance change value of road network under emergency.

\begin{tabular}{|c|c|}
\hline Arcs & $\Delta L(h)$ \\
\hline 1 & 0.499829543 \\
\hline 2 & 0.517159174 \\
\hline 3 & 0.506221114 \\
\hline 4 & 0.511633593 \\
\hline 5 & 0.526580431 \\
\hline 6 & 0.488086389 \\
\hline 7 & 0.479349968 \\
\hline 8 & 0.513842596 \\
\hline 9 & 0.498912608 \\
\hline 10 & 0.50785149 \\
\hline 11 & 0.508517681 \\
\hline 12 & 0.52126487 \\
\hline 13 & 0.496948847 \\
\hline 14 & 0.491859617 \\
\hline 15 & 0.497418344 \\
\hline 16 & 0.486644673 \\
\hline 17 & 0.483751788 \\
\hline 18 & 0.479275825 \\
\hline 19 & 0.497191832 \\
\hline 20 & 0.50924756 \\
\hline 21 & 0.5004505 \\
\hline 22 & 0.4893875 \\
\hline 23 & 0.5279817 \\
\hline 24 & 0.5117103 \\
\hline 25 & 0.5251609 \\
\hline 26 & 0.5162785 \\
\hline 27 & 0.4788009 \\
\hline 28 & 0.4833101 \\
\hline 29 & 0.4913568 \\
\hline 30 & 0.5049395 \\
\hline 31 & 0.486535 \\
\hline 32 & 0.471266 \\
\hline 33 & 0.498022 \\
\hline 34 & 0.505899 \\
\hline 35 & 0.511477 \\
\hline 36 & 0.469642 \\
\hline 37 & 0.476885 \\
\hline 38 & 0.487584 \\
\hline 39 & 0.462275 \\
\hline 40 & 0.488921 \\
\hline 41 & 0.505747 \\
\hline 42 & 0.512273 \\
\hline 43 & 0.510029 \\
\hline 44 & 0.521001 \\
\hline 45 & 0.490435 \\
\hline 46 & 0.506765 \\
\hline 47 & 0.490421 \\
\hline 48 & 0.489485 \\
\hline 49 & 0.480163 \\
\hline 50 & 0.495179 \\
\hline 51 & 0.503999 \\
\hline 52 & 0.496315 \\
\hline 53 & 0.500324 \\
\hline 54 & 0.467583 \\
\hline 55 & 0.516451 \\
\hline 56 & 0.499224 \\
\hline 57 & 0.50053 \\
\hline 58 & 0.473564 \\
\hline 59 & 0.487039 \\
\hline
\end{tabular}

$(\Delta E)$ caused by these nodes were large, indicating that these nodes and related road sections had high vulnerability.

\subsection{Analysis of Vulnerability Identification Results considering} Connectivity, Network Efficiency, and Traffic Performance. The vulnerability of the road network under the change of road traffic demand was identified, Table 8 shows the results. However, in the actual operation of the urban road network, when an emergency occurs, it would not only lead to a change of road traffic demand, but also to that of the road network structure. Therefore, it was necessary to comprehensively consider the changes of road network connectivity and efficiency as well as the traffic performance when analyzing the traffic VURN under emergency conditions. Based on this, the vulnerability of the road network was comprehensively identified.

In Section 3.1, "Index Design," the vulnerability comprehensive identification method based on road network connectivity and efficiency as well as traffic performance were discussed, and the vulnerability comprehensive identification index including road network connectivity, efficiency, and traffic performance was designed $(v)$ (see equation (7)). In this equation, three model parameters were involved, namely, $\varepsilon, \varnothing$, and $\gamma$, and the other three model variables are the road network connectivity change value $\left(\Delta G^{\prime}\right)$, network efficiency change value $\left(\Delta E^{\prime}\right)$, and traffic performance change value $\left(\Delta L^{\prime}\right)$ after data standardization. Among them, the model parameters required specific calibration. Here, the Delphi method was used for this purpose. Through the investigation of 15 transportation experts, parameter calibration results were obtained, and the parameters $\varepsilon, \varnothing$, and $\gamma$ were $0.42,0.23$. and 0.35 , respectively. By substituting them into equation (7), the following results were obtained:

$$
v=0.42 \Delta G^{\prime}+0.23 \Delta E^{\prime}+0.35 \Delta L^{\prime} .
$$

Since the change value of network efficiency $\left(\Delta e^{\prime}\right)$ and the change value of traffic performance $\left(\Delta L^{\prime}\right)$ were based on the road section, to unify into equation (26), it was necessary to convert the change value of road network connectivity $\left(\Delta G^{\prime}\right)$ based on the node to the road section and subsequently calculate on this basis to obtain the results shown in Table 10 .

According to the scale range of the vulnerability level of key nodes or sections of the road network set in Table 1, the corresponding vulnerability level was given in the last column as shown in Table 10. Among them, the vulnerability level of road section 12 was "high," sections $23,37,11$, and 24 was "medium," and the remaining sections were below "low." Figure 8 shows the statistical chart of some data, and Figure 9 shows the distribution of the vulnerability level in the road network.

Compared with Tables 9 and 10, the former only considered the changes of network connectivity and network efficiency under emergency conditions. This distribution only reflected the vulnerability of a static road network, 


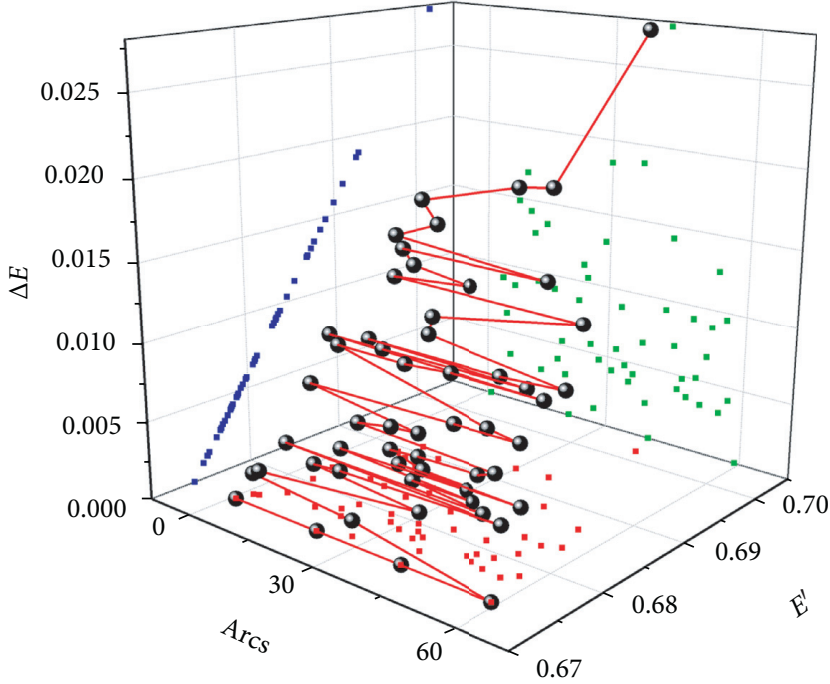

\begin{tabular}{|l|c|c|}
\hline Arcs & $E^{\prime}$ & $\Delta E$ \\
\hline 42 & 0.701 & 0.027 \\
\hline 37 & 0.693 & 0.018 \\
\hline 30 & 0.692 & 0.018 \\
\hline 10 & 0.691 & 0.016 \\
\hline 16 & 0.69 & 0.015 \\
\hline 8 & 0.689 & 0.014 \\
\hline 11 & 0.688 & 0.014 \\
\hline 44 & 0.688 & 0.014 \\
\hline 15 & 0.687 & 0.013 \\
\hline 29 & 0.687 & 0.013 \\
\hline 12 & 0.686 & 0.012 \\
\hline 54 & 0.686 & 0.012 \\
\hline 24 & 0.685 & 0.011 \\
\hline 25 & 0.684 & 0.010 \\
\hline- & - & - \\
\hline
\end{tabular}

Vulnerability ranking of arc segments

- Arcs

Figure 6: Road network vulnerability identification based on efficiency.

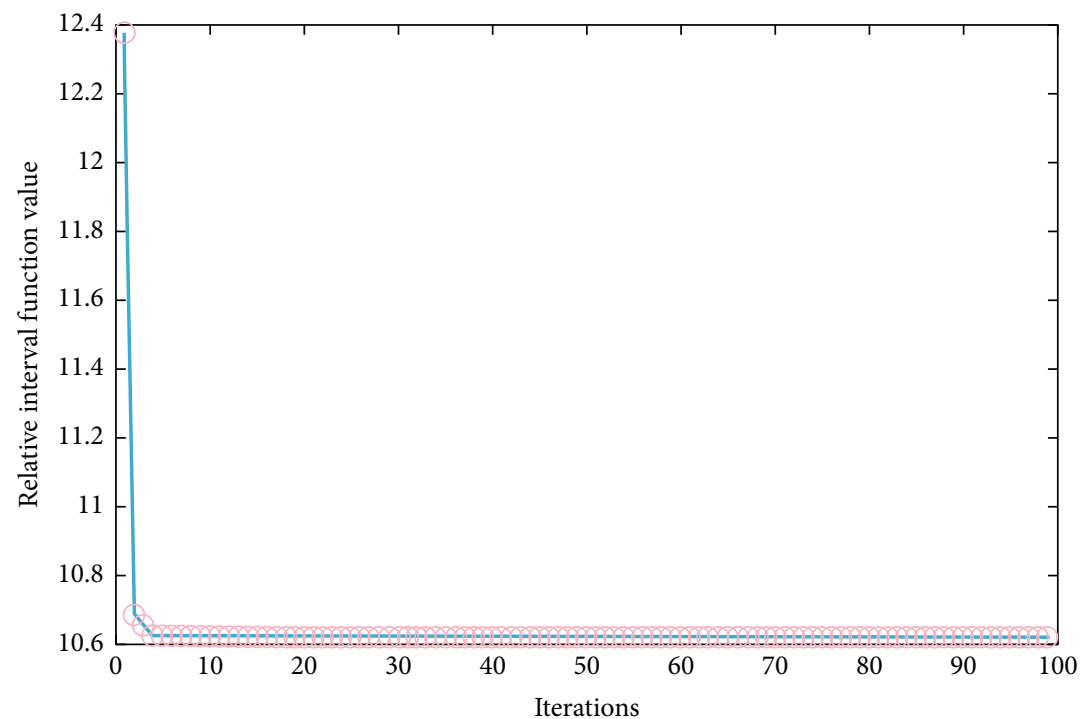

FIGURE 7: Convergence of the algorithm.

TABLE 9: Vulnerability identification results based on network connectivity and network efficiency (partial data).

\begin{tabular}{|c|c|c|c|c|c|}
\hline \multicolumn{2}{|c|}{ Network connectivity } & \multicolumn{4}{|c|}{ Network efficiency } \\
\hline Nodes & Connectivity change $(\Delta G)$ & Nodes & Nodes & Road section & Network efficiency change $(\Delta E)$ \\
\hline 9 & 0.033459596 & 22 & 24 & 42 & 0.0277 \\
\hline 11 & 0.033459596 & 13 & 25 & 37 & 0.0188 \\
\hline 24 & 0.033459596 & 6 & 11 & 30 & 0.0185 \\
\hline 25 & 0.033459596 & 15 & 18 & 10 & 0.0169 \\
\hline 26 & 0.033459596 & 9 & 10 & 16 & 0.0158 \\
\hline 6 & 0.023042929 & 1 & 15 & 8 & 0.0148 \\
\hline 12 & 0.023042929 & 14 & 15 & 11 & 0.0142 \\
\hline 13 & 0.023042929 & 24 & 25 & 44 & 0.0142 \\
\hline 15 & 0.023042929 & 6 & 7 & 15 & 0.0135 \\
\hline 16 & 0.023042929 & 5 & 6 & 29 & 0.0131 \\
\hline
\end{tabular}


TABle 10: Comprehensive identification results of road network vulnerability based on connectivity, network efficiency, and traffic performance.

\begin{tabular}{|c|c|c|c|c|c|}
\hline Arcs & $\Delta G^{\prime}$ & $\Delta E^{\prime}$ & $\Delta L^{\prime}$ & $v$ & Vulnerability level \\
\hline 12 & 1 & 0.458484 & 0.897775 & 0.839672622 & 4 \\
\hline 23 & 0.666656 & 0.310469 & 1 & 0.701403462 & 3 \\
\hline 37 & 1 & 0.6787 & 0.222352 & 0.653924174 & 3 \\
\hline 11 & 0.666656 & 0.512635 & 0.703774 & 0.64422256 & 3 \\
\hline 24 & 0.666656 & 0.404332 & 0.752363 & 0.63631881 & 3 \\
\hline 38 & 1 & 0.238267 & 0.385181 & 0.609614902 & 2 \\
\hline 15 & 0.666656 & 0.487365 & 0.534852 & 0.579287444 & 2 \\
\hline 33 & 0.666656 & 0.31769 & 0.544039 & 0.543477668 & 2 \\
\hline 5 & 0.333312 & 0.231047 & 0.978674 & 0.535667593 & 2 \\
\hline 10 & 0.333312 & 0.610108 & 0.693635 & 0.523088253 & 2 \\
\hline 27 & 1 & 0.036101 & 0.25151 & 0.516331787 & 2 \\
\hline 36 & 1 & 0.151625 & 0.112119 & 0.494115447 & 1 \\
\hline 17 & 0.666656 & 0.32491 & 0.326858 & 0.469125175 & 1 \\
\hline 49 & 0.666656 & 0.32852 & 0.27224 & 0.450839102 & 1 \\
\hline 2 & 0.333312 & 0.050542 & 0.83529 & 0.443967135 & 1 \\
\hline 53 & 0.333312 & 0.31769 & 0.579073 & 0.415735252 & 1 \\
\hline 56 & 0.333312 & 0.34296 & 0.562332 & 0.415688156 & 1 \\
\hline 18 & 0.666656 & 0.140794 & 0.258738 & 0.402936491 & 1 \\
\hline 13 & 0.333312 & 0.33574 & 0.527706 & 0.401908448 & 1 \\
\hline 16 & 0.333312 & 0.570397 & 0.370886 & 0.40099232 & 1 \\
\hline 3 & 0.333312 & 0.057762 & 0.668822 & 0.387364017 & 1 \\
\hline 1 & 0.333312 & 0 & 0.571548 & 0.340032836 & 1 \\
\hline 4 & 0 & 0.32491 & 0.751196 & 0.337647674 & 1 \\
\hline 48 & 0.333312 & 0.129964 & 0.414113 & 0.314822252 & 1 \\
\hline 31 & 0.333312 & 0.148014 & 0.369216 & 0.303260111 & 1 \\
\hline 40 & 0.333312 & 0 & 0.405529 & 0.281926296 & 1 \\
\hline 54 & 0.333312 & 0.454874 & 0.080783 & 0.272886104 & 1 \\
\hline 46 & 0 & 0.144404 & 0.6771 & 0.270197893 & 1 \\
\hline 14 & 0 & 0.101083 & 0.450253 & 0.180837486 & 1 \\
\hline 39 & 0 & 0.086643 & 0 & 0.019927798 & 1 \\
\hline
\end{tabular}

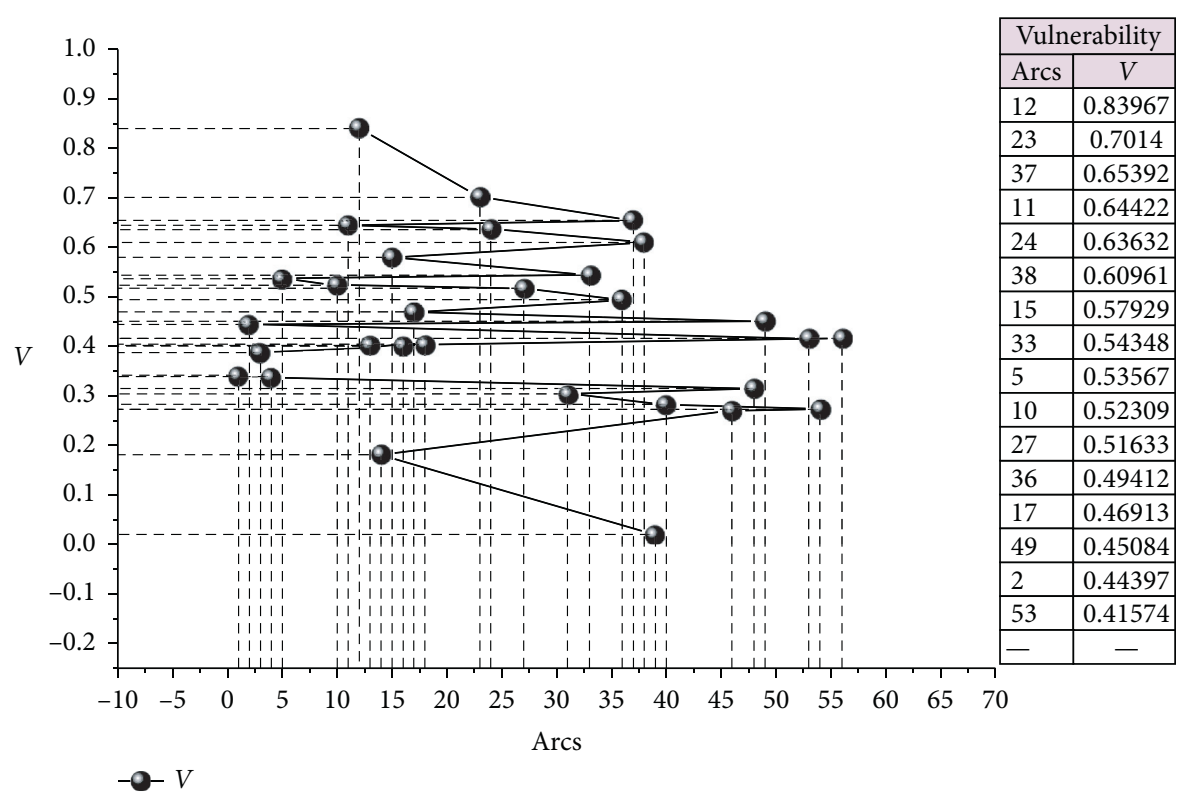

FIGURE 8: Vulnerability statistics after comprehensive identification.

including the connectivity of road sections in the network topology and the change of network efficiency characterized by the shortest path distribution. The latter did not consider the changes of static structure to the network under emergency conditions, nor did it consider the changes of the same in the network due to the accidents that change traffic 


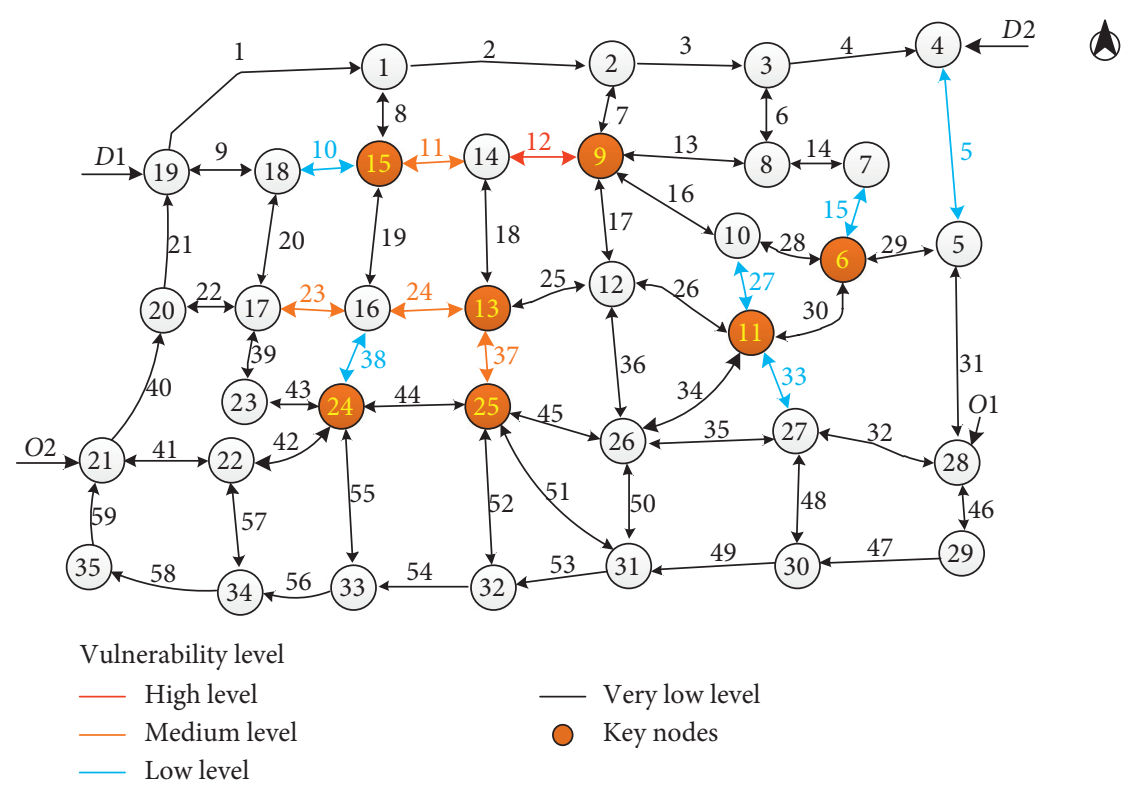

FIGURE 9: Vulnerability level display.

performance (change to traffic impedance of the road network) caused by the change in network traffic demand (change of road users' travel choice) was closer to the real road traffic situation.

Due different considerations, there was variation in the results of different discriminant indexes. From the identification results, only considering the changes of network connectivity and efficiency under emergency conditions, it was concluded that the vulnerability of nodes $9,11,24,25,6$, and 13 and that of road sections $7,12,37,24,16$, and 17 were relatively large. If the changes of road network connectivity and efficiency as well as the traffic performance were considered simultaneously, it was concluded that the vulnerability of sections 12 , $23,37,11,24,38$, and 15 was relatively high. Compared with the others, road sections 12 and 37 were considered two sections with greater vulnerability. Therefore, the two indicators had certain directivity. From another point of view, they also showed that sections 12 and 37 should be considered two sections with outstanding vulnerability. In the daily maintenance and after the occurrence of the emergency, these sections and nodes were the key links that departments needed to focus on. The comprehensive identification of road network traffic vulnerability based on a variety of discriminant indicators could represent the vulnerability from different angles, and various discriminant indicators could complement each other.

In the identification of urban road network traffic vulnerability, considering the network structure and traffic operation conditions under emergency conditions, the conclusion is closer to that of the actual road traffic situation. However, this comprehensive identification method based on multiple evaluation indexes was also limited by the model accuracy and operation efficiency. At present, room for improvement remains in the application of the proposed solution based on multiple discriminant indicators proposed in this study in terms of solving the large-scale vulnerability identification and evaluation.

\section{Conclusions}

The accurate, effective, and reliable identification of traffic VURNs can provide scientific guidance for road traffic planning, design, construction, operation, management, evaluation, and emergency rescue. Although the academic community has contributed much research into this, there is no unified and definite definition for the concept of road traffic vulnerability. Because of different interpretations regarding this, the research ideas proposed are dissimilar. Based on summarizing the existing research results, we proposed three core identification indexes from the perspectives of static road network structure and dynamic traffic performance, and a comprehensive identification index of urban road network traffic vulnerability based on this. Hence, the vulnerability identification model of the urban road traffic performance index and its solution algorithm were studied. Finally, to verify the designed vulnerability identification method, taking the road network of Nanshan Science and Technology Park area in Shenzhen City as an example, the single vulnerability identification index and comprehensive vulnerability identification index of an urban road network were verified, respectively. The results demonstrated that the proposed vulnerability comprehensive evaluation index had greater advantages than a single type of vulnerability evaluation index, because there was better complementarity between them.

The results of this study could be applied in the following contexts. First, it could be used to guide road management departments in recognizing the vulnerable links of the traffic system, assist in determining the priority of road maintenance and repair, and reduce the vulnerability of important traffic infrastructure. Second, it is conducive to the need to optimize the operation and management of daily traffic networks and reduce traffic congestion and time delay. Third, it is beneficial to evaluate the potential consequences 
and overall effects of various control and management strategies and enhance the ability to prevent and respond to disasters and emergency situations. Fourth, it is beneficial to evaluate the connectivity vulnerability of various road network planning schemes and enhance the prevention and response capabilities through traffic network planning.

Because research on the vulnerability of urban road networks under emergency conditions involves too many uncertain factors, including engineering, technical, economic, social, and other aspects of urban development, it was difficult to provide a definitive solution. However, future work will concentrate on the vulnerability of key coupling networks in an urban multimode traffic network, with a focus on improving the practical application of the research.

\section{Data Availability}

The raw data supporting the research of this article will be made available to any qualified researcher by the authors.

\section{Conflicts of Interest}

The author declares no conflicts of interest.

\section{Authors' Contributions}

$\mathrm{H}$. Xiang conceived the research project and proposed the algorithm and conducted the experiments.

\section{Acknowledgments}

This article was partially supported by the Science and Technology Projects of Shenzhen Science and Technology Innovation Committee (no. JSGG20170822093602485) and Shenzhen Fundamental Research Project (no. JCYJ20180305163701198). The author would like to thank Editage (https://www.editage.cn) for English language editing.

\section{References}

[1] A. D'Andrea, S. Cafiso, and A. Condorelli, "Methodological considerations for the evaluation of seismic risk on road network," Pure and Applied Geophysics, vol. 162, no. 4, pp. 767-782, 2005.

[2] B. Y. Chen, W. H. K. Lam, A. Sumalee, Q. Li, and Z.-C. Li, "Vulnerability analysis for large-scale and congested road networks with demand uncertainty," Transportation Research Part A: Policy and Practice, vol. 46, no. 3, pp. 501-516, 2012.

[3] C.-H. Hsieh and C.-M. Feng, "The highway resilience and vulnerability in Taiwan," Transport Policy, vol. 87, pp. 1-9, 2020.

[4] I. Cambridge Systematics, An Initial Assessment of Freight Bottlenecks on Highways, Federal Highway Administration, U.S. transportation of Department, Washington, DC, USA, 2005, https://www.fhwa.dot.gov/policy/otps/bottlenecks/.

[5] A. Nagurney, Q. Qiang, and L. S. Nagurney, "Environmental impact assessment of transportation networks with degradable links in an era of climate change," International Journal of Sustainable Transportation, vol. 4, no. 3, pp. 154-171, 2010.
[6] K. Berdica, "An introduction to road vulnerability: what has been done, is done and should be done," Transport Policy, vol. 9, no. 2, pp. 117-127, 2002.

[7] F. Rupi, S. Bernardi, G. Rossi, and A. Danesi, "The evaluation of road network vulnerability in mountainous areas: a case study," Networks and Spatial Economics, vol. 15, no. 2, pp. 397-411, 2015.

[8] L. Gao, X. Liu, Y. Liu et al., "Measuring road network topology vulnerability by Ricci curvature," Physica A: Statistical Mechanics and Its Applications, vol. 527, Article ID 121071, 2019.

[9] E. Jenelius, T. Petersen, and L.-G. Mattsson, "Importance and exposure in road network vulnerability analysis," Transportation Research Part A, vol. 40, no. 7, 2005.

[10] Z. Zheng, Z. Huang, F. Zhang, and P. Wang, "Understanding coupling dynamics of public transportation networks," EPJ Data Science, vol. 7, no. 1, 2018.

[11] M. Weber, E. Saucan, and J. Jost, "Characterizing complex networks with forman-ricci curvature and associated geometric flows," Journal of Complex Networks, vol. 5, no. 4, pp. 527-550, 2017.

[12] M. Li, H. Wei, Y. Li, and S. Liu, "Identifying influential nodes in complex networks based on local and global methods," Journal of Physics: Conference Series, vol. 1738, no. 1, 2021.

[13] Y. Duan and F. Lu, "Robustness of city road networks at different granularities," Physica A: Statistical Mechanics and Its Applications, vol. 411, pp. 21-34, 2014.

[14] D.-B. Chen, H. Gao, L. Lü, and T. Zhou, "Identifying influential nodes in large-scale directed networks: the role of clustering," PLoS One, vol. 8, no. 10, Article ID e77455, 2013.

[15] H.-Y. Yin and X. U. Li-Qun, "A model for identifying vulnerable links of road networks based on bayesian networks," Journal of Systems\& Management, vol. 19, no. 6, pp. 656-661, 2010, in Chinese.

[16] E. L. D. Oliveira, L. D. S. Portugal, and W. P. Junior, "Determining critical links in a road network: vulnerability and congestion indicators," Procedia-Social and Behavioral Sciences, vol. 162, pp. 158-167, 2014.

[17] S. Jung, S. Lee, O. Kwon, and B. Kim, "Grid-based traffic vulnerability analysis by using betweenness centrality," Journal of the Korean Physical Society, vol. 77, no. 7, pp. 538-544, 2020.

[18] R. Milo, S. Itzkovitz, N. Kashtan et al., "Superfamilies of evolved and designed networks," Science, vol. 303, no. 5663, 2004.

[19] M. Snelder, H. J. Van Zuylen, and L. H. Immers, "A framework for robustness analysis of road networks for short term variations in supply," Transportation Research Part A: Policy and Practice, vol. 46, no. 5, pp. 828-842, 2012.

[20] D. M. Scott, D. C. Novak, L. Aultman-Hall, and F. Guo, "Network robustness index: a new method for identifying critical links and evaluating the performance of transportation networks," Journal of Transport Geography, vol. 14, no. 3, pp. 215-227, 2006.

[21] Z.-P. Du and A. Nicholson, "Degradable transportation systems: sensitivity and reliability analysis," Transportation Research Part B: Methodological, vol. 31, no. 3, pp. 225-237, 1997.

[22] A. Chen, H. Yang, H. K. Lo, and W. H. Tang, "Capacity reliability of a road network: an assessment methodology and numerical results," Transportation Research Part B: Methodological, vol. 36, no. 3, pp. 225-252, 2002.

[23] E. Jenelius, T. Petersen, and L.-G. Mattsson, "Importance and exposure in road network vulnerability analysis," 
Transportation Research Part A: Policy and Practice, vol. 40, no. 7, pp. 537-560, 2006.

[24] J. Sohn, "Evaluating the significance of highway network links under the flood damage: an accessibility approach," Transportation Research Part A: Policy and Practice, vol. 40, no. 6, pp. 491-506, 2006.

[25] M. A. P. Taylor, S. V. C. Sekhar, and G. M. D’Este, “Application of accessibility based methods for vulnerability analysis of strategic road networks," Networks and Spatial Economics, vol. 6, no. 3-4, pp. 267-291, 2006.

[26] K. Berdica and L. G. Mattsson, "Vulnerability: a modelasedcase study of the road network in stockholm," Critical Infrastructure: Reliability and Vulnerability, A. T. Burray and T. H. Grubesic, Eds., Springer, Berlin, Germany, 2007.

[27] A. Chen, C. Yang, S. Kongsomsaksakul, and M. Lee, "Network-based accessibility measures for vulnerability analysis of degradable transportation networks," Networks and Spatial Economics, vol. 7, no. 3, pp. 241-256, 2007.

[28] L.-G. Mattsson and E. Jenelius, "Vulnerability and resilience of transport systems - a discussion of recent research," Transportation Research Part A: Policy and Practice, vol. 81, pp. 16-34, 2015.

[29] A. J. Holmgren, "A framework for vulnerability assessment of electric power systems," Reliability and Vulnerability in Critical Infrastructure: A Quantitative Geographic Perspective, A. Murray and T. Grubesic, Eds., Springer, Berlin, Germany, 2007.

[30] J. Husdal, "The vulnerability of road networks in a cost-benefit perspective," 2005.

[31] M. A. P. Taylor and G. M. D’Este, “Transport network vulnerability: a method for diagnosis of critical locations in transport infrastructure systems," Critical Infrastructure: Reliability and Vulnerability, A. T. Murray and T. H. Grubesic, Eds., Springer, Berlin, Germany, 2007.

[32] A. Erath, J. Birdsall, K. W. Axhausen et al., "Vulnerability assessment of the swiss road network," in Proceedings of the 88th Transportation Research Board Annual Meeting, pp. 1-17, Washington DC, USA, March 2009.

[33] E. Jenelius and L.-G. Mattsson, "Road network vulnerability analysis: conceptualization, implementation and application," Computers, Environment and Urban Systems, vol. 49, pp. 136-147, 2015.

[34] Y. Lu-Ping and D.-L. Qian, "Vulnerability analysis of road networks," Journal of Transportation Systems Engineering and Information Technology, vol. 12, no. 2, pp. 105-110, 2011, in Chinese.

[35] M. Lujak and S. Giordani, "Centrality measures for evacuation: finding agile evacuation routes," Future Generation Computer Systems, vol. 83, pp. 401-412, 2018.

[36] United Nations International Strategy for Disaster Reduction, Living with Risk: A Global Review of Disaster Reduction Initiatives, United NationsInternational Strategy for Disaster Reduction, Geneva, Switzerland, 2004.

[37] Y. Liu, "Emergency response facility location in transportation networks: a literature review," Journal of Traffic and Transportation Engineering, vol. 3, 2021, English Edition.

[38] R. Z. Farahani, M. M. Lotfi, A. Baghaian et al., "Mass casualty management in disaster scene: a systematic review of OR\&MS research in humanitarian operations," European Journal of Operational Research, vol. 287, no. 3, Article ID 787e819, 2020.

[39] A. P. Mera and C. Balijepalli, "Towards improving resilience of cities: an optimisation approach to minimising vulnerability to disruption due to natural disasters under budgetary constraints," Transportation, vol. 47, no. 4, pp. 1809-1842, 2020.

[40] F. Makoto, "A study of vulnerability of emergency transport road network to various hazards," Građevinar, vol. 70, no. 12, pp. 1065-1074, 2018.

[41] H. Zhang and Y. Yao, "An integrative vulnerability evaluation model to urban road complex network," Wireless Personal Communications, vol. 107, no. 1, pp. 193-204, 2019.

[42] A. B. Morelli and A. L. Cunha, "Measuring urban road network vulnerability to extreme events: an application for urban floods," Transportation Research Part D: Transport and Environment, vol. 93, Article ID 102770, 2021.

[43] J. Liu, Z. Shi, and X. Tan, "Measuring the dynamic evolution of road network vulnerability to floods: a case study of Wuhan, China," Travel Behaviour and Society, vol. 23, pp. 13-24, 2021.

[44] M. Ansari Esfeh, A. E. Salari, L. Kattan, W. H. K. Lam, R. Ansari Esfe, and M. Salari, "Compound generalized extreme value distribution for modeling the effects of monthly and seasonal variation on the extreme travel delays for vulnerability analysis of road network," Transportation Research Part C: Emerging Technologies, vol. 120, Article ID 102808, 2020.

[45] M. M. De Brito, M. Evers, and B. Höllermann, "Prioritization of flood vulnerability, coping capacity and exposure indicators through the Delphi technique: a case study in TaquariAntas basin, Brazil," International Journal of Disaster Risk Reduction, vol. 24, pp. 119-128, 2017.

[46] L. Jun-Qiang, A. Zhai Jing, L. Qian-Wen, and L. Zhao, "Construction of road network vulnerability evaluation index based on general travel cost," Physica A, vol. 493, pp. 421-429, 2018.

[47] N. He and S. Zhao, "Discussion on influencing factors of free-flow travel time in road traffic impedance function," Procedia - Social and Behavioral Sciences, vol. 96, pp. 90-97, 2013.

[48] T. P. Van Oijen, W. Daamen, and S. P. Hoogendoorn, "Estimation of a recursive link-based logit model and link flows in a sensor equipped network," Transportation Research Part B: Methodological, vol. 140, pp. 262-281, 2020. 